\title{
HITTING AND ESCAPING STATISTICS: MIXING, TARGETS AND HOLES
}

\author{
HENK BRUIN, MARK F. DEMERS, AND MIKE TODD
}

\begin{abstract}
There is a natural connection between two types of recurrence law: hitting times to shrinking targets, and hitting times to a fixed target (usually seen as escape through a hole). We show that for systems which mix exponentially fast, one can move through a natural parameter space from one to the other. On the other hand, if the mixing is subexponential, there is a phase transition between the hitting times law and the escape law.
\end{abstract}

\section{INTRODUCTION}

This work is motivated by the natural connection between escape rates and hitting times. The existence of an exponential Hitting Time Statistics (HTS) law, which is a recurrence law to shrinking targets, is a rather soft condition: in all cases we are aware of, all one requires is mixing, with no rates necessary. However, under some mixing conditions, good error bounds can be derived (see e.g. [FFT2]) which mean that we can change the scaling in that law and still derive a non-degenerate limit law. If the mixing is exponential, the scaling can be changed to recover an escape rate to a fixed hole/target. In this paper we explore a parameter space which takes us between the escape rate case and the hitting time case. Under exponential mixing we can go between these laws in a non-degenerate way. A phase transition occurs when one leaves the hitting time setting and heads towards the escape case whenever the system is subexponentially mixing. In this paper we address such transitions in the case of stretched exponential, super-polynomial and polynomial rates of mixing.

1.1. Hitting times, escape rates, and between. Given a dynamical system $f: X \circlearrowleft$ preserving an ergodic probability measure $\mu$, one can consider first entry times to a sequence of subsets $\left(U_{r}\right)_{r}$ with $U_{r}$ shrinking to a given point $z$ as $r \rightarrow 0$. Letting $\tau_{r}$ be the first hitting time to $U_{r}$, i.e.,

$$
\tau_{r}(x):=\inf \left\{n \geqslant 1: f^{n}(x) \in U_{r}\right\},
$$

one can ask how the quantity $\mu\left(\tau_{r}>t\right)$ depends asymptotically on both $r$ and $t$ (for a fixed $z$ ). To derive a HTS law, one scales the time via $t=s / \mu\left(U_{r}\right)$ for some $s \in \mathbb{R}^{+}$and considers the

Date: December 19, 2017.

2000 Mathematics Subject Classification. 37A25, 37C30, 37E05, 37D35, 37D25.

Key words and phrases. Hitting time statistics; open systems; escape rates; recurrence; mixing rates.

MD was partially supported by NSF grant DMS 1362420. This project was started as part of an RIG grant through ICMS, Scotland. The authors would like to thank ICMS for its generous hospitality. They would also like to thank the ICERM Semester Program on Dimension and Dynamics and the ESI Thematic Programme Mixing Flows and Averaging Methods where part of this work was carried out. 
limit

$$
\lim _{r \rightarrow 0} \mu\left(\tau_{r}>s / \mu\left(U_{r}\right)\right)
$$

For a large range of dynamical systems it is known that this limit is $e^{-s}$ for $\mu$-a.e. centre $z$. So we obtain an expression which is more convenient in this work:

$$
\lim _{r \rightarrow 0}-\frac{1}{s} \log \mu\left(\tau_{r}>s / \mu\left(U_{r}\right)\right)=1 \quad \text { for } \mu \text {-a.e. } z \text {. }
$$

There is a wealth of literature on this topic, but here we just refer to the reviews $[\mathrm{H}]$ and $\left[\mathrm{LF}^{+}\right.$, Chapter 5] and note that we only require very basic mixing properties for (1.1); for example, for multimodal maps of the interval, if there is an absolutely continuous invariant measure (with no mixing requirement), this law holds $[\mathrm{BT}]$.

From the point of view of open systems, one declares $U_{r}$ to be a (fixed) hole and considers any point entering $U_{r}$ to be annihilated from the system. In contrast to hitting times (where $\tau_{r}(x) \geq 1$ a.s. also for $\left.x \in U_{r}\right)$, in an open system a point $x \in U_{r}$ is not allowed to exit $U_{r}$. Thus the escape time $e_{r}(x)$ satisfies $e_{r}(x)=\tau_{r}(x)$ if $x \notin U_{r}$ and $e_{r}(x)=0$ if $x \in U_{r}$. However, an essential connection between the two is given by,

$$
\left\{x \in X: \tau_{r}(x)=t\right\}=f^{-1}\left(\left\{x \in X: e_{r}(x)=t-1\right\}\right), \quad \text { for all } t \geqslant 1 .
$$

Due to the invariance of $\mu$, the escape rate can be defined by the following equivalent expressions,

$$
\lim _{t \rightarrow \infty}-\frac{1}{t} \log \mu\left(e_{r}>t-1\right)=\lim _{t \rightarrow \infty}-\frac{1}{t} \log \mu\left(\tau_{r}>t\right)
$$

when this limit exists. If the limit exists, we label it $-\log \lambda_{r}$ for reasons that will become clear later and consider the 'derivative of the escape rate', expressed as the limit,

$$
\lim _{r \rightarrow 0} \frac{-\log \lambda_{r}}{\mu\left(U_{r}\right)}=1 \quad \text { for } \mu \text {-a.e. } z,
$$

which has been proved for certain exponentially mixing systems BY, KL2. We are not aware of examples where the limit in (1.2) exists (in the exponentially mixing setting), but (1.3) fails. Naturally if the system is subexponentially mixing, then 1.2 should be degenerate and so 1.3 . fails, see [DF]. One expects (see e.g. [FP, [FFT1]) that for periodic points $z$, the limit will be some number in $(0,1)$ which can be expressed in terms of the relevant potential; if $f$ is continuous, for all other points the limit should be 1 . The recent work [PU] extends this point of view to a wide variety of conformal systems via symbolic dynamics.

Both of the limits (1.1) and (1.3) can be seen as special limiting cases of the expression

$$
\frac{1}{\mu\left(U_{r}\right)} \frac{-1}{t} \log \mu\left(\tau_{r}>t\right)
$$

where the open system perspective takes first the limit $t \rightarrow \infty$ then $r \rightarrow 0$, while the hitting time perspective takes the 'diagonal limit' $r \rightarrow 0$ with $t=s / \mu\left(U_{r}\right)$.

Once one views this expression in the two-dimensional parameter space $(r, t)$, one can naturally ask questions regarding convergence along various paths through this parameter space. Setting $t=s \mu\left(U_{r}\right)^{-\alpha}$ for some $\alpha, s \in(0, \infty)$, we formulate the generalised limit

$$
L_{\alpha, s}(z):=\lim _{r \rightarrow 0} \frac{-1}{s \mu\left(U_{r}\right)^{1-\alpha}} \log \mu\left(\tau_{r}>s \mu\left(U_{r}\right)^{-\alpha}\right),
$$


if the limit exists. With this formulation, the case $\alpha=1$ coincides with the diagonal limit formulated above for hitting time statistics. Additionally, $\alpha=\infty$ can be thought of as coinciding with the derivative of the escape rate (1.3) (where $t \rightarrow \infty$ as $r$ is held fixed), while $\alpha=0$ can be thought of as the reversed order of limits,

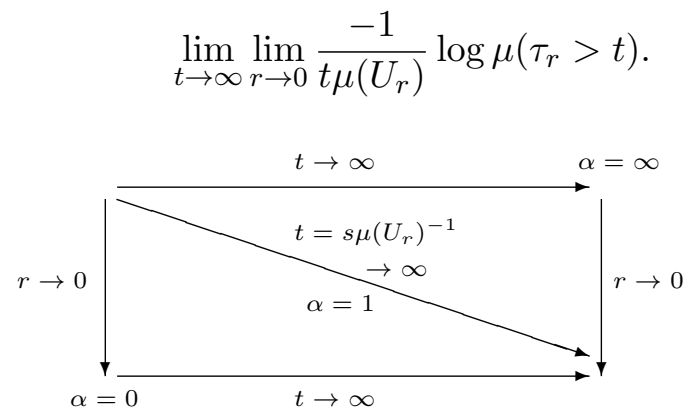

FiguRE 1. Different paths of taking the limit $r \rightarrow 0, t \rightarrow \infty$, with $t=s \mu\left(U_{r}\right)^{-\alpha}$.

Remark 1.1. For every $\alpha \in[0,1)$, and supposing $\mu\left(\tau_{r}>t\right)>0$ for all $t, L_{\alpha, s}(z) \in[0,1]$, provided it exists. Indeed, for $t=s \mu\left(U_{r}\right)^{-\alpha}$, we have

$$
\begin{aligned}
0 & \leqslant \frac{-\log \mu\left(\tau_{r}>t\right)}{s \mu\left(U_{r}\right)^{1-\alpha}}=\frac{-\log \left(1-\mu\left(\tau_{r} \leqslant t\right)\right)}{s \mu\left(U_{r}\right)^{1-\alpha}} \\
& =\frac{-\log \left(1-\mu\left(\cup_{j=0}^{t-1} f^{-j}\left(U_{r}\right)\right)\right)}{s \mu\left(U_{r}\right)^{1-\alpha}} \leqslant \frac{-\log \left(1-t \mu\left(U_{r}\right)\right)}{s \mu\left(U_{r}\right)^{1-\alpha}} \\
& =\frac{-\log \left(1-s \mu\left(U_{r}\right)^{1-\alpha}\right)}{s \mu\left(U_{r}\right)^{1-\alpha}} \rightarrow 1 \quad \text { as } \mu\left(U_{r}\right) \rightarrow 0 .
\end{aligned}
$$

Therefore, any limit point belongs to $[0,1]$. The calculation above also implies that, when the limit exists, for $\alpha<1$,

$$
\lim _{r \rightarrow 0} \frac{-\log \mu\left(\tau_{r}>s \mu\left(U_{r}\right)^{-\alpha}\right)}{s \mu\left(U_{r}\right)^{1-\alpha}}=\lim _{r \rightarrow 0} \frac{\mu\left(\tau_{r} \leqslant s \mu\left(U_{r}\right)^{-\alpha}\right)}{s \mu\left(U_{r}\right)^{1-\alpha}} .
$$

1.2. Brief summary of results. Our main results are, roughly speaking, that if the system behaves well and is exponentially mixing, then $L_{\alpha, s}(z)$ exists for all $\alpha$; it can be written in terms of the periodic behaviour if $z$ is periodic, and $L_{\alpha, s}(z)=1$ otherwise (Theorem 2.1). On the other hand, if the system is slower than stretched exponentially mixing then $L_{\alpha, s}(z)=0$ for $\alpha>1$. If the system is (exactly) stretched exponentially mixing then there exists an $\alpha_{0}>1$ depending on the mixing rate so that we have the same result as for the exponential case if $\alpha<\alpha_{0}$, and $L_{\alpha, s}(z)=0$ for $\alpha>\alpha_{0}$ (Theorem 3.2). The latter results employ an inducing argument and a large deviations law (either exponential, stretched exponential or polynomial). Our examples using inducing schemes require good large deviations of the inducing time, with various types of tail.

We remark that the existence of $L_{\alpha, s}(z)$ for $\alpha \neq 1$ is more delicate than for $\alpha=1$ and gives additional information about the distribution of $\tau_{r}$. For example, in the generic case, when $\alpha=1$, one obtains that $\mu\left(\tau_{r}>s \mu\left(U_{r}\right)^{-1}\right) \rightarrow e^{-s}$ as $r \rightarrow 0$, but the rate of convergence does not appear. By contrast, when $\alpha \neq 1$, the limit of $\mu\left(\tau_{r}>s \mu\left(U_{r}\right)^{-\alpha}\right)$ is always either 1 (for $\alpha<1$ ) or 0 (for $\alpha>1$ ), and $L_{\alpha, s}(z)$ captures the exponential rate at which this convergence occurs. This 
rate provides information about the tail distribution of $\tau_{r}$ for small $r$. Again using the generic case as an example, when $\alpha>1, L_{\alpha, s}(z)=1$ implies $\mu\left(\tau_{r}>s \mu\left(U_{r}\right)^{-\alpha}\right)=e^{-(1 \pm \varepsilon) s \mu\left(U_{r}\right)^{1-\alpha}}$; when $\alpha<1, L_{\alpha, s}(z)=1$ implies $\mu\left(\tau_{r} \leqslant s \mu\left(U_{r}\right)^{-\alpha}\right)=(1 \pm \varepsilon) s \mu\left(U_{r}\right)^{1-\alpha}$, due to (1.6).

The paper is organised as follows. In Section 2, we consider interval maps with good spectral properties; namely, that an associated family of transfer operators has a spectral gap. Our results are formulated abstractly, but in Section 2.6 we give specific examples including LasotaYorke maps, Gibbs-Markov maps and the Gauss map. In Section 3, we consider systems where a well-chosen first return map has good properties and show how the tail of the first return time affects the limits $L_{\alpha, s}$. Again we formulate our results abstractly and then provide examples in Section 4 to a variety of maps, including generalised Farey maps, several classes of unimodal maps and Young towers. In the appendix we (re)prove two technical results used in Sections 2 and 3 .

Notation. We will use the following notational conventions throughout the paper without further mention. $A=C^{ \pm 1} B$ means there exists $C \geqslant 1$ such that $C^{-1} B \leqslant A \leqslant C B$; similarly, $A=(1 \pm \varepsilon) B$ means $(1-\varepsilon) B \leqslant A \leqslant(1+\varepsilon) B$. We write $A \sim B$ if $\lim A / B=1$, where the parameter in which the limit is taken is clear by context (usually it is $r \rightarrow 0$ ).

\section{Exponentially MiXing CASE}

In this section, we consider a piecewise continuous map of the unit interval $f: I \circlearrowleft$, with countably many intervals of monotonicity. Our assumptions will be general enough to allow both traditional piecewise expanding maps as well as more general Gibbs-Markov maps with contracting potentials. We will then prove our results regarding $L_{\alpha, s}(z)$, defined in (1.5), with respect to equilibrium states for these potentials. We will make assumptions on the map ((F1)(F4) below as well as (U), and where appropriate $(\mathbf{P})$ ) which imply the conditions of Rychlik $[\mathrm{R}]$ as well as giving a form of the Lasota-Yorke inequality needed in Proposition 2.5.

Assume that there exists a countable collection of maximal intervals $\mathcal{Z}=\left\{Z_{i}\right\}_{i}, Z_{i} \subset I$, with disjoint interiors, such that $f$ is continuous and strictly monotonic on each $Z_{i}$. We set $D=$ $I \backslash \cup_{i} \operatorname{int}\left(Z_{i}\right)$.

We assume that there exists a (nonatomic) Borel probability measure $m_{\varphi}$ such that $m_{\varphi}(D)=0$, which is conformal with respect to a potential $\varphi: I \rightarrow \mathbb{R}$, i.e., $d m_{\varphi} / d\left(m_{\varphi} \circ f\right)=e^{\varphi}$. The associated transfer operator acting on $L^{1}\left(m_{\varphi}\right)$ is

$$
\mathcal{L}_{\varphi} \psi(x)=\sum_{y \in f^{-1} x} \psi(y) e^{\varphi(y)}, \quad \forall \psi \in L^{1}\left(m_{\varphi}\right) .
$$

We will study the action of $\mathcal{L}_{\varphi}$ on functions of bounded variation. To that end, recall that the variation of a function $\psi$ on an interval $J$ is defined by

$$
\bigvee_{J} \psi=\sup \left\{\sum_{i=0}^{k-1}\left|\psi\left(x_{i+1}\right)-\psi\left(x_{i}\right)\right|: x_{0}<x_{1}<\cdots<x_{k}, x_{i} \in J, \forall i \leqslant k\right\},
$$

where the supremum is taken over all finite sets $\left\{x_{i}\right\}_{i} \subset J$. 
Let $S_{n} \varphi=\sum_{i=0}^{n-1} \varphi \circ f^{i}$. We set $\left.\varphi\right|_{D}=-\infty$ and assume the potential $\varphi$ satisfies the following regularity properties:

(F1) $\exists C_{d}>0$ s.t. $\left|e^{S_{n} \varphi(x)-S_{n} \varphi(y)}-1\right| \leqslant C_{d}\left|f^{n} x-f^{n} y\right|$ whenever $f^{i} x, f^{i} y$ lie in the same element of $\mathcal{Z}$ for $i=0,1, \ldots, n-1$;

(F2) $\sum_{Z \in \mathcal{Z}} \sup _{Z} e^{\varphi}<\infty$;

(F3) $\exists n_{0} \in \mathbb{N}$ such that $\sup _{I} e^{S_{n_{0}} \varphi}<\inf _{I \backslash D} \mathcal{L}_{\varphi}^{n_{0}} 1$;

(F4) for each interval $J \subset I \backslash D, \exists N=N(J)$ s.t. $\inf _{I \backslash D} \mathcal{L}_{\varphi}^{N} 1_{J}>0$, where $1_{J}$ is the indicator function of the set $J$.

Due to the existence of the conformal measure $m_{\varphi}$, we have $\int \mathcal{L}_{\varphi}^{n} 1 d m_{\varphi}=\int 1 d m_{\varphi}=1$, so

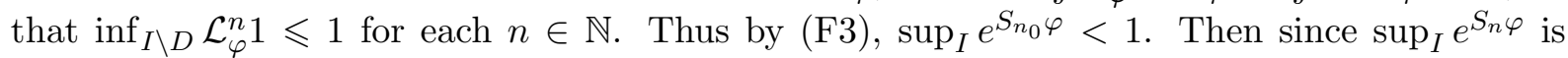
submultiplicative,

$$
\exists n_{1} \in \mathbb{N} \text { such that }\left(2+2 C_{d}\right) \sup _{I} e^{S_{n_{1}} \varphi}<1,
$$

where $C_{d}$ is from $(\mathrm{F} 1)$.

Now fix $z \in I$ and for $r_{0}>0$, define $\left(U_{r}\right)_{r \in\left(0, r_{0}\right)}$ to be a family of intervals such that $\operatorname{diam}\left(U_{r}\right) \rightarrow 0$ as $r \rightarrow 0$, and $\cap_{r} U_{r}=\{z\}$. From the point of view of open systems, for each $r$, we define the map with hole $U_{r}$ and its iterates by, $\stackrel{\circ}{r}_{r}^{n}=\left.f^{n}\right|_{i_{r}^{n-1}}$, where $\stackrel{\circ}{I}_{r}^{0}=I \backslash U_{r}$ and $\stackrel{\circ}{I}_{r}^{n}=\cap_{i=0}^{n} f^{-i}\left(I \backslash U_{r}\right)$.

Let $\mathcal{I}_{r}^{n}$ denote the intervals of monotonicity for $f_{r}^{n}$. We assume the following uniform large images condition for $f_{r}^{n_{1}}$ on the sequence $\left(U_{r}\right)_{r}$.

(U) There exists $c_{0}>0$ such that

$$
\inf _{r \in\left[0, r_{0}\right]} \inf \left\{m_{\varphi}\left(\AA_{r}^{n_{1}} J\right): J \in \mathcal{I}_{r}^{n_{1}}\right\} \geqslant c_{0},
$$

where $n_{1}$ is from (2.1).

As we shall show in Section 2.1, under assumptions (F1)-(F4), $\mathcal{L}_{\varphi}$ admits a unique invariant measure $\mu_{\varphi}$, absolutely continuous with respect to $m_{\varphi}$, whose density $g_{0}$ is of bounded variation and is bounded away from 0 . Note that $\mu_{\varphi}$ can also be characterised as an equilibrium state for $\varphi$. That is, for the variational pressure $P(\varphi):=\sup \left\{h(\mu)+\int \varphi d \mu\right\}$ where the supremum is taken over all $f$-invariant probability measures, $\mu_{\varphi}$ is an equilibrium state since it satisfies $h\left(\mu_{\varphi}\right)+\int \varphi d \mu_{\varphi}=P(\varphi)$. Moreover, since we can also think of $P(\varphi)$ as the log of the leading eigenvalue of $\mathcal{L}_{\varphi}$, our assumptions here give $P(\varphi)=0$.

In the case when $z$ is periodic of prime period $p$, we shall need the following additional condition.

(P) The density $g_{0}$ is continuous at $z$ and $f^{p}$ is monotonic at $z$.

Let

$$
I_{\text {cont }}:=\left\{z \in I: f^{k} \text { is continuous at } z \text { for all } k \in \mathbb{N}\right\} .
$$

That $\mu_{\varphi}\left(I_{\text {cont }}\right)=1$ follows from the assumption that $m_{\varphi}(D)=0$. The main result of this section is the following theorem. 
Theorem 2.1. Let $(f, \varphi)$ satisfy $(F 1)-(F 4)$. Fix $z \in I_{\text {cont }}$ and let $\left(U_{r}\right)_{r \in\left(0, r_{0}\right]}$ be a family of intervals such that $\lim _{r \rightarrow 0} \operatorname{diam}\left(U_{r}\right)=0$ and $\cap_{r} U_{r}=\{z\}$, satisfying $(\mathbf{U})$, and in the periodic case, $\mathbf{( P )}$ as well.

Then, for any $s \in \mathbb{R}^{+}$and $\alpha \in[0, \infty]$, taking $L_{\alpha, s}(z)$ with respect to the invariant measure $\mu_{\varphi}$, we have

$$
L_{\alpha, s}(z)= \begin{cases}1, & \text { if } z \text { is not periodic } \\ 1-e^{S_{p} \varphi(z)}, & \text { if } z \text { is p-periodic, }\end{cases}
$$

where $p$-periodic means that the prime period of $z$ is $p$.

Remark 2.2. If $f$ is continuous, then $I=I_{\text {cont }}$, while in the context of hitting time statistics, the case $z \in I \backslash I_{\text {cont }} \neq \emptyset$ is addressed in [AFV, Section 3.3].

Remark 2.3. As will be clear from the proof of Theorem 2.1, the case $\alpha=0$ holds in great generality: The proof in Section 2.4 requires neither (F2)-(F4) nor (U). In the non-periodic case we require only that $z \in I_{\text {cont }}$. In the periodic case, we require $(F 1),(\mathbf{P})$ and the fact that $\mu_{\varphi}$ is absolutely continuous with respect to $m_{\varphi}$ with density bounded away from 0 at $z$. Alternatively, if it is known that $e^{S_{p} \varphi}$ is continuous at $z$, then (F1) is not needed.

2.1. Preliminaries. We begin by establishing some easy facts about the potential $\varphi$. Let $\mathcal{Z}^{n}=\bigvee_{i=0}^{n-1} f^{-i}(\mathcal{Z})$ denote the maximal intervals on which $f^{n}$ is continuous and monotonic.

Lemma 2.4. Assuming (F1)-(F4), for all $n \geqslant 0$, the following hold:

(a) $\sum_{Z \in \mathcal{Z}^{n}} e^{S_{n} \varphi}<\infty$;

(b) for each $Z \in \mathcal{Z}, \bigvee_{Z} e^{\varphi} \leqslant C_{d} \sup _{Z} e^{\varphi}$;

(c) $\bigvee_{I} e^{S_{n} \varphi}<\infty$.

Proof. (a) follows from a standard inductive argument using (F2).

(b) follows from (F1) since $\left|e^{\varphi\left(x_{i+1}\right)}-e^{\varphi\left(x_{i}\right)}\right| \leqslant C_{d} e^{\varphi\left(x_{i}\right)}$ for any set $\left\{x_{i}\right\}_{i=1}^{k} \subset Z$.

For $n=0$, (c) follows from (b) and (F2). Note that setting $\left.\varphi\right|_{D}=-\infty$ only adds a term bounded by the series in (F2) to the variation. For $n \geqslant 1$, the argument again follows from a standard induction.

Potentials satisfying the above properties in addition to (F3) are called contracting potentials in the literature (see for example, [R, [LSV1]), while (F4) is called the covering property. However, we require (F1) in order to obtain the stronger form of Lasota-Yorke inequalities in Proposition 2.5, which we shall need to apply perturbation theory to the open systems $\left(\AA_{r}, U_{r}\right)$, considering $U_{r}$ as a hole 11 We will prove Theorem 2.1 using the fact that the transfer operators associated with both the closed and open systems have spectral gaps and their spectral projectors vary in some uniform way with the size of the hole.

Let $\mathcal{B}$ be the set of functions of bounded variation on $I$ equipped with the variation norm $\|\psi\|=\bigvee_{I} \psi+|\psi|_{1}$, where $|\cdot|_{1}$ denotes the $L^{1}$-norm with respect to the conformal measure $m_{\varphi}$.

\footnotetext{
${ }^{1}$ For a way to relax condition (F1) by requiring only a Hölder bound on distortion, see the application of Theorem 2.1 to the Gauss map in Section 2.6.3.
} 
Using Lemma 2.4 and (2.1), the operator $\mathcal{L}_{\varphi}^{n_{1}}$ satisfies the assumptions of [R, Theorem 1], so that $\mathcal{L}_{\varphi}^{n_{1}}$ is quasi-compact as an operator on $\mathcal{B}$. Now using the decomposition in $\mathbb{R}$, Theorem 3 ] and the covering property (F4), it follows that $\mathcal{L}_{\varphi}^{n_{1}}$ has a simple eigenvalue at 1 , and no other eigenvalue can have modulus 1, i.e., $\mathcal{L}_{\varphi}^{n_{1}}$ has a spectral gap. Using again Lemma 2.4(b),(c), since $\mathcal{L}_{\varphi}$ is a bounded operator on $\mathcal{B}$, it also has a spectral gap. This will be the starting point from which we will perturb.

2.2. Notation and Initial Results for Open Systems. In this section, we first summarise standard notation for open systems that we will use throughout the paper. We then proceed to prove the existence of a uniform spectral gap for a family of associated transfer operators.

Recall that if we regard $U_{r}$ as a hole, then the set of points that has not entered $U_{r}$ by time $n$ is denoted $\stackrel{\circ}{I}_{r}^{n}=\cap_{i=0}^{n} f^{-i}\left(I \backslash U_{r}\right)$, and the map corresponding to the open system is simply the restriction $f_{r}^{n}:=\left.f^{n}\right|_{I_{r}^{n-1}}$. Notice that by definition of the escape time $e_{r}$ (see Section 1.1), we have $\left\{e_{r}>n\right\}=\stackrel{\circ}{I}_{r}^{n}$.

The transfer operator for the open system and its iterates are defined for $\psi \in L^{1}\left(m_{\varphi}\right)$ by

$$
\stackrel{\circ}{\mathcal{L}}_{\varphi, U_{r}}^{n} \psi(x)=\sum_{y \in \dot{f}_{r}^{-n} x} \psi(y) e^{S_{n} \varphi(y)}=\mathcal{L}_{\varphi}^{n}\left(\psi 1_{I_{r}^{n-1}}\right),
$$

for all $n \geqslant 1$. Due to the conformality of the measure $m_{\varphi}$, we have the following useful identity,

$$
\int_{I} \stackrel{\circ}{\mathcal{L}}_{\varphi, U_{r}}^{n} \psi d m_{\varphi}=\int_{I} \mathcal{L}_{\varphi}^{n}\left(\psi 1_{\check{I}_{r}^{-n-1}}\right) d m_{\varphi}=\int_{\check{I}_{r}^{n-1}} \psi d m_{\varphi}
$$

The importance of the above relation is the connection it provides between the escape rate with respect to the measure $\psi d m_{\varphi}$ and the spectral radius of $\stackrel{\circ}{\mathcal{L}}_{\varphi, U_{r}}$ acting on functions of bounded variation (see Proposition 2.5).

Since we fix the potential $\varphi$, for ease of notation and to emphasise the relationships among the operators, in what follows we will denote $\stackrel{\circ}{\mathcal{L}}_{r}:=\stackrel{\circ}{\mathcal{L}}_{\varphi, U_{r}}$ and $\mathcal{L}_{0}:=\mathcal{L}_{\varphi}$. Similarly, we denote by $g_{0}$ the invariant density for $\mathcal{L}_{0}, m_{0}$ the conformal measure, and $\mu_{0}=g_{0} m_{0}$ the invariant measure for the closed system.

Due to $(\mathbf{U})$, we have the following set of uniform Lasota-Yorke inequalities for this family of operators.

Proposition 2.5. There exists $C_{0}>0$ and $\sigma<1$ such that for any $\psi \in \mathcal{B}, r \in\left[0, r_{0}\right]$ and all $n \geq 0$,

$$
\begin{aligned}
\left\|\stackrel{\mathcal{L}}{r}_{r}^{n} \psi\right\| & \leqslant C_{0} \sigma^{-n}\|\psi\|+C_{0} \int_{\check{I}_{r}^{n-1}}|\psi| d m_{0}, \\
\left|\dot{\mathcal{L}}_{r}^{n} \psi\right|_{1} & \leqslant \int_{\check{I}_{r}^{n-1}}|\psi| d m_{0} .
\end{aligned}
$$

The proof is by now fairly standard, even in this generalised context. Since our assumptions and estimates necessarily differ from those appearing in the literature for closed systems (given that we must show uniformity of the constants $C_{0}$ and $\sigma$ in the sequence $\left(U_{r}\right)$ as well as the fact that we require decay in the $L^{1}$ term), we include the proof for completeness in the appendix. 
It follows from Proposition 2.5. the compactness of the unit ball of $\mathcal{B}$ in $L^{1}\left(m_{0}\right)$, and the conformality of $m_{0}$ that the spectral radius of $\dot{\mathcal{L}}_{r}$ acting in $\mathcal{B}$ is at most one while its essential spectral radius is bounded by $\sigma^{-1}<1$. Thus $\dot{\mathcal{L}}_{r}$ is quasi-compact as an operator on $\mathcal{B}$, as is $\mathcal{L}_{0}$. In addition, defining the following perturbative norm,

$$
||\left|\mathcal{L}_{0}-\dot{\mathcal{L}}_{r}\right| \|=\sup \left\{\left|\mathcal{L}_{0} \psi-\dot{\mathcal{L}}_{r} \psi\right|_{1}:\|\psi\| \leqslant 1\right\},
$$

we have the following bound.

Lemma 2.6. ||$\left|\mathcal{L}_{0}-\stackrel{\circ}{\mathcal{L}}_{r}\right| \| \leqslant m_{0}\left(U_{r}\right) \leqslant C_{1} \mu_{0}\left(U_{r}\right)$, where $C_{1}^{-1}=$ essinf $g_{0}$.

Proof. The proof is immediate since if $\psi \in \mathcal{B}$ with $\|\psi\| \leqslant 1$, we use the fact that $m_{0}$ is $\varphi$ conformal to estimate,

$$
\int\left|\left(\mathcal{L}_{0}-\dot{\mathcal{L}}_{r}\right) \psi\right| d m_{0}=\int\left|\mathcal{L}_{0}\left(1_{U_{r}} \psi\right)\right| d m_{0} \leqslant|\psi|_{\infty} m_{0}\left(U_{r}\right),
$$

and the fact that essinf $g_{0}>0$ follows from (F4).

Corollary 2.7. There exists $r_{1} \in\left(0, r_{0}\right]$ such that for all $r \in\left[0, r_{1}\right]$, the operators $\stackrel{\mathcal{L}}{r}_{r}$ have a uniform spectral gap on $\mathcal{B}$. In particular, for $r>0$, there exist $\lambda_{r}<1$, and linear operators $\Pi_{r}$, $R_{r}$, such that

$$
\stackrel{\circ}{\mathcal{L}}_{r}=\lambda_{r} \Pi_{r}+R_{r},
$$

$\Pi_{r}^{2}=\Pi_{r}, \Pi_{r} R_{r}=R_{r} \Pi_{r}=0$ and the spectral radius of $R_{r}$ is at most $\rho<\inf \left\{\lambda_{r}: r<r_{1}\right\}$. The range of $\Pi_{r}$ is the span of a function $g_{r} \in \mathcal{B}$, satisfying $\stackrel{\circ}{\mathcal{L}}_{r} g_{r}=\lambda_{r} g_{r}$, and normalised so that $\int g_{r} d m_{0}=1$.

The above decomposition also holds for $r=0$ with $\lambda_{0}=1$.

Proof. $\mathcal{L}_{0}$ has a spectral gap by $[\mathrm{R}]$ and the discussion following Lemma 2.4. It follows from Proposition 2.5. Lemma 2.6 and [KL1, Corollary 1] that the spectra and spectral projectors of $\dot{\mathcal{L}}_{r}$ and $\mathcal{L}_{0}$ outside the disk of radius $\sigma$ vary continuously in $\mu_{0}\left(U_{r}\right)$. Thus for $r$ sufficiently small, $\mathcal{L}_{r}$ inherits a spectral gap from $\mathcal{L}_{0}$, and by continuity, the spectral gap is uniform in $r$, yielding the existence of $\rho<\inf \left\{\lambda_{r}: r<r_{1}\right\}$ in the statement of the corollary.

We proceed to the proof of Theorem 2.1. first proving the special cases $\alpha=\infty$ and $\alpha=0$, and then turning to the general case $\alpha \in(0, \infty)$.

2.3. Proof of Theorem 2.1: The case $\alpha=\infty$. To address the case corresponding to $\alpha=\infty$, we must compute the double limit,

$$
\lim _{r \rightarrow 0} \lim _{t \rightarrow \infty} \frac{1}{\mu_{0}\left(U_{r}\right)} \frac{-1}{t} \log \mu_{0}\left(\tau_{r}>t\right) .
$$

For fixed $r \in\left(0, r_{1}\right]$, the spectral gap provided by Corollary 2.7 implies that the escape rate with respect to $\mu_{0}$ is $-\log \lambda_{r}$, i.e.,

$$
\lim _{t \rightarrow \infty} \frac{1}{t} \log \mu_{0}\left(\tau_{r}>t\right)=\lim _{t \rightarrow \infty} \frac{1}{t} \log \mu_{0}\left(\stackrel{I}{r}_{r}^{t}\right)=\log \lambda_{r},
$$

where we have used $(1.2)$ as well as the fact that $\left\{e_{r}>t\right\}=I_{r}^{t}$. (Indeed, the escape rate is $-\log \lambda_{r}$ with respect to the measure $\psi m_{0}$ for any density $\psi \in \mathcal{B}$ that is bounded away from 0 .) 
In order to show the limit $r \rightarrow 0$ converges to the claimed value, we will use the results of [KL2]. To do this we must check the necessary conditions given there, listed as (A1)-(A7). In our setting, (A1)-(A3) are immediately satisfied by the existence of a uniform spectral gap for the operators $\mathcal{L}_{r}$ and the accompanying spectral decomposition given by Corollary 2.7 .

(A4) requires that we normalise $m_{0}\left(g_{r}\right)=1$, which we have done, and that there exists $C_{2}>0$ such that $\sup _{r \in\left[0, r_{1}\right]}\left\|g_{r}\right\| \leqslant C_{2}$, i.e., the conditionally invariant densities are uniformly bounded in $\mathcal{B}$. This follows from the uniform Lasota-Yorke inequalities given by Proposition 2.5 applied to $g_{r}$ :

$$
\lambda_{r}^{n}\left\|g_{r}\right\|=\left\|\dot{\mathcal{L}}_{r}^{n} g_{r}\right\| \leqslant C_{0} \sigma^{n}\left\|g_{r}\right\|+C_{0} \int_{\dot{I}_{r}^{n}} g_{r} d m_{0}=C_{0} \sigma^{n}\left\|g_{r}\right\|+C_{0} \lambda_{r}^{n}
$$

where we have used the fact that $\int_{\check{I}_{r}^{n}} g_{r} d m_{0}=\int{\stackrel{\circ}{\mathcal{L}_{r}^{n}}}_{r} g_{r} d m_{0}$, by conformality. Since $\sigma<\lambda_{r}$ in the spectral gap regime, we let $n \rightarrow \infty$ and conclude that $\left\|g_{r}\right\| \leqslant C_{0}$ independently of $r \in\left[0, r_{1}\right]$.

(A5) requires that $\eta_{r}:=\left\|m_{0}\left(\mathcal{L}_{0}-\stackrel{\circ}{\mathcal{L}}_{r}\right)\right\| \rightarrow 0$ as $r \rightarrow 0$, where $\|\cdot\|$ is the norm of the linear functional $m_{0}\left(\mathcal{L}_{0}-\dot{\mathcal{L}}_{r}\right): \mathcal{B} \rightarrow \mathbb{R}$. This is precisely Lemma 2.6, since if $\psi \in \mathcal{B}$, we have

$$
\left|m_{0}\left(\left(\mathcal{L}_{0}-\stackrel{\circ}{\mathcal{L}}_{r}\right)(\psi)\right)\right| \leqslant \int\left|\left(\mathcal{L}_{0}-\stackrel{\circ}{\mathcal{L}}_{r}\right) \psi\right| d m_{0} \leqslant\|\psi\| m_{0}\left(U_{r}\right)
$$

so that $\eta_{r}=m_{0}\left(U_{r}\right)$.

(A6) requires ${ }^{2} \eta_{r} \cdot\left\|\left(\mathcal{L}_{0}-\stackrel{\circ}{\mathcal{L}}_{r}\right) g_{0}\right\| \leqslant C_{3} \mu_{0}\left(U_{r}\right)$, for some $C_{3}>0$. This is satisfied since (as noted in Lemma 2.6), essinf $g_{0}=C_{1}^{-1}>0$. Thus,

$$
\begin{aligned}
\eta_{r} \cdot\left\|\left(\mathcal{L}_{0}-\dot{\mathcal{L}}_{r}\right) g_{0}\right\| & =m_{0}\left(U_{r}\right)\left\|\mathcal{L}_{0}\left(1_{U_{r}} g_{0}\right)\right\| \leqslant C_{1} \mu_{0}\left(U_{r}\right)\left\|\mathcal{L}_{0}\right\|\left\|1_{U_{r}} g_{0}\right\| \\
& \leqslant C_{1}\left\|\mathcal{L}_{0}\right\|\left(\left\|g_{0}\right\|+2\left|g_{0}\right|_{\infty}\right) \mu_{0}\left(U_{r}\right) \leqslant 3 C_{1} C_{0}\left\|\mathcal{L}_{0}\right\| \mu_{0}\left(U_{r}\right),
\end{aligned}
$$

as required.

Finally, (A7) requires that the limit

$$
q_{k}:=\lim _{r \rightarrow 0} q_{k, r}:=\lim _{r \rightarrow 0} \frac{m_{0}\left(\left(\mathcal{L}_{0}-\stackrel{\circ}{\mathcal{L}}_{r}\right) \stackrel{\circ}{\mathcal{L}}_{r}^{k}\left(\mathcal{L}_{0}-\stackrel{\circ}{\mathcal{L}}_{r}\right)\left(g_{0}\right)\right)}{\mu_{0}\left(U_{r}\right)},
$$

exists for each integer $k \geqslant 0$. Notice that by conformality and using the fact that $\mathcal{L}_{0}-\dot{\mathcal{L}}_{r}=$ $\mathcal{L}_{0}\left(1_{U_{r}} \cdot\right)$, we have

$$
m_{0}\left(\left(\mathcal{L}_{0}-\stackrel{\circ}{\mathcal{L}}_{r}\right) \stackrel{\circ}{\mathcal{L}}_{r}^{k}\left(\mathcal{L}_{0}-\stackrel{\circ}{\mathcal{L}}_{r}\right)\left(g_{0}\right)\right)=\int 1_{U_{r}} \circ f^{k+1} \cdot 1_{\check{I}_{r}^{k-1}} \circ f \cdot 1_{U_{r}} \cdot g_{0} d m_{0} .
$$

The product of indicator functions in the above expression is equivalent to the indicator function of the set

$$
E_{r}^{k}=\left\{x \in U_{r}: f^{i}(x) \notin U_{r}, i=1, \ldots, k, \text { and } f^{k+1}(x) \in U_{r}\right\} .
$$

So $q_{k, r}=\frac{\mu_{0}\left(E_{r}^{k}\right)}{\mu_{0}\left(U_{r}\right)}$.

\footnotetext{
${ }^{2}$ KL2 actually states this bound, and subsequent ones, in terms of a more general quantity, $\Delta_{r}$; however, in the present context, $\Delta_{r}=\int\left(\mathcal{L}_{0}-\stackrel{\bullet}{\mathcal{L}}_{r}\right) g_{0} d m_{0}=\int_{U_{r}} g_{0} d m_{0}=\mu_{0}\left(U_{r}\right)$, and we will use this simpler expression in what follows.
} 
If $z$ is not periodic, recall that since $z \in I_{\text {cont }}, f^{k}$ is continuous at $z$ for each $k \in \mathbb{N}$, so for fixed $k$, the set $E_{r}^{k}$ is empty for all $r$ sufficiently small. Thus $q_{k}=0$ for all $k \geqslant 0$. On the other hand, if $z$ is periodic with prime period $p$, then for sufficiently small $r, E_{r}^{k}$ is empty except when $k=p-1$. In this case, we use the monotonicity and continuity of $f^{p}$ at $z$ (assumption $(\mathbf{P})$ ) to conclude that $f^{p}\left(E_{r}^{p-1}\right)=U_{r}$. Let $f_{1}$ denote this branch of $f^{p}$. Now the continuity of $g_{0}$ at $z$ and (F1) yield,

$$
q_{p-1}=\lim _{r \rightarrow 0} \frac{1}{\mu_{0}\left(U_{r}\right)} \int_{E_{r}^{p-1}} g_{0} d m_{0}=\lim _{r \rightarrow 0} \frac{1}{\mu_{0}\left(U_{r}\right)} \int_{U_{r}} e^{S_{p} \varphi \circ f_{1}^{-p}} g_{0} \circ f_{1}^{-p} d m_{0}=e^{S_{p} \varphi(z)},
$$

where we have used the fact that $f_{1}^{-p}(z)=z$.

Having verified conditions (A1)-(A7) in our setting, we conclude by [KL2, Theorem 2.1], that for $z \in I_{\text {cont }}$,

$$
\lim _{r \rightarrow 0} \frac{-1}{\mu_{0}\left(U_{r}\right)} \log \lambda_{r}= \begin{cases}1, & \text { if } z \text { is not periodic } \\ 1-e^{S_{p} \varphi(z)}, & \text { if } z \text { is } p \text {-periodic. }\end{cases}
$$

2.4. Proof of Theorem 2.1; The case $\alpha=0$. Since we will not need the measures $\mu_{r}$ in this section, for simplicity, we will denote $\mu_{0}$ simply by $\mu$ and $m_{0}$ by $m$.

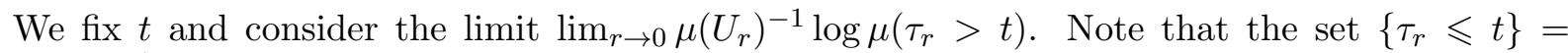
$\cup_{j=0}^{t} f^{-j}\left(U_{r}\right)$.

Case 1: Nonperiodic $z$. Assume that $z \in I_{\text {cont }}$ is not a periodic point for $f$. Then we may choose $r$ sufficiently small that the sets $f^{-j}\left(U_{r}\right), j=0, \ldots, t$, are pairwise disjoint. Thus,

$$
\begin{aligned}
\lim _{r \rightarrow 0} \mu\left(U_{r}\right)^{-1} \log \mu\left(\tau_{r}>t\right) & =\lim _{r \rightarrow 0} \mu\left(U_{r}\right)^{-1} \log \left(1-\mu\left(\tau_{r} \leqslant t\right)\right) \\
& =\lim _{r \rightarrow 0} \mu\left(U_{r}\right)^{-1} \log \left(1-(t+1) \mu\left(U_{r}\right)\right)=-(t+1) .
\end{aligned}
$$

Proceeding to the second limit, we complete the proof of this case,

$$
\lim _{t \rightarrow \infty} \lim _{r \rightarrow 0}-t^{-1} \mu\left(U_{r}\right)^{-1} \log \mu\left(\tau_{r}>t\right)=\lim _{t \rightarrow \infty} t^{-1}(t+1)=1 .
$$

Case 2: Periodic z. Fix $z$ of prime period $p$ for $f$, satisfying $(\mathbf{P})$. Choose $r$ sufficiently small that the sets $f^{-i}\left(U_{r}\right), i=0, \ldots, p-1$ are pairwise disjoint. This choice forces $f^{-i}\left(U_{r}\right) \cap f^{-j}\left(U_{r}\right)=\emptyset$ except when $i-j$ is a multiple of $p$.

Suppose $t \in \mathbb{N}$ satisfies $t=(k+1) p-1$ for some $k \geqslant 0$. Then

$$
\left\{\tau_{r} \leqslant t\right\}=\cup_{i=0}^{k} \cup_{j=0}^{p-1} f^{-i p-j}\left(U_{r}\right) .
$$

Note that by the above observation regarding when two pre-images of $U_{r}$ may intersect, we conclude that the sets in the union above are disjoint for distinct $j$. Thus,

$$
\mu\left(\tau_{r} \leqslant t\right)=\sum_{j=0}^{p-1} \mu\left(\cup_{i=0}^{k} f^{-i p-j}\left(U_{r}\right)\right)=p \mu\left(\cup_{i=0}^{k} f^{-i p}\left(U_{r}\right)\right) .
$$

To estimate the measure of the remaining set, we prove the following lemma. 
Lemma 2.8. Let $z$ be a point of continuity of $g_{0}$ of prime period $p$. For $\varepsilon>0$, let $U_{r}$ be a sufficiently small neighbourhood of $z$ with $\operatorname{diam}\left(U_{r}\right)<\varepsilon$ such that $f^{p}$ is monotonic on $U_{r}$ and for each $x \in U_{r},\left|g_{0}(x)-g_{0}(z)\right| \leqslant \varepsilon$. If $k$ is such that $U_{r}, U_{r} \cap f^{-p}\left(U_{r}\right), \ldots, U_{r} \cap f^{-k p}\left(U_{r}\right)$ forms a decreasing sequence of sets, then

$$
\mu\left(\cup_{i=0}^{k} f^{-i p}\left(U_{r}\right)\right)=\mu\left(U_{r}\right)\left(k+1-k e^{S_{p} \varphi(z)}(1 \pm \bar{C} \varepsilon)\right),
$$

where $\bar{C}=C_{d}+C_{1}, C_{d}$ is from (F1) and $C_{1}$ is from Lemma 2.6.

Proof. Write $V_{r}=U_{r} \cap f^{-p}\left(U_{r}\right)$. The proof goes by induction on $k$. For $k=1$, we have

$$
\begin{aligned}
\mu\left(U_{r} \cup f^{-p}\left(U_{r}\right)\right) & =\mu\left(U_{r}\right)+\mu\left(f^{-p}\left(U_{r}\right)\right)-\mu\left(V_{r}\right) \\
& =2 \mu\left(U_{r}\right)-\mu\left(U_{r} \cap f^{-p}\left(U_{r}\right)\right) .
\end{aligned}
$$

Letting $f_{1}^{p}$ denote the branch of $f^{p}$ mapping $V_{r}$ onto $U_{r}$ monotonically,

$$
\begin{aligned}
\mu\left(V_{r}\right)= & \int_{U_{r} \cap f^{-p}\left(U_{r}\right)} g_{0} d m=\int_{U_{r}} g_{0} \circ f_{1}^{-p} e^{S_{n} \varphi \circ f_{1}^{-p}} d m \\
= & \mu\left(U_{r}\right) e^{S_{p} \varphi(z)}+\int_{U_{r}} g_{0} \circ f_{1}^{-p} e^{S_{p} \varphi \circ f_{1}^{-p}}\left(1-e^{S_{p} \varphi(z)-S_{p} \varphi \circ f_{1}^{-p}}\right) d m \\
& \quad+e^{S_{p} \varphi(z)} \int_{U_{r}}\left(g_{0} \circ f_{1}^{-p}-g_{0}\right) d m
\end{aligned}
$$

Using (F1), the first integral on the right hand side is bounded by

$$
e^{S_{p} \varphi(z)} C_{d} \operatorname{diam}\left(U_{r}\right) \int_{U_{r}} g_{0} \circ f_{1}^{-p} e^{S_{p} \varphi \circ f_{1}^{-p}} d m \leqslant e^{S_{p} \varphi(z)} C_{d} \operatorname{diam}\left(U_{r}\right) \mu\left(U_{r}\right),
$$

where we have changed variables again for the last inequality. The second integral on the right hand side is bounded by,

$$
e^{S_{p} \varphi(z)} \varepsilon m\left(U_{r}\right) \leqslant e^{S_{p} \varphi(z)} C_{1} \varepsilon \mu\left(U_{r}\right),
$$

where $C_{1}$ is from Lemma 2.6. Putting these estimates together and using the fact that $\operatorname{diam}\left(U_{r}\right)<$ $\varepsilon$, we obtain,

$$
\mu\left(V_{r}\right)=(1 \pm \bar{C} \varepsilon) e^{S_{p} \varphi(z)} \mu\left(U_{r}\right)
$$

where $\bar{C}=C_{d}+C_{1}$.

Plugging this into (2.6) yields the lemma for $k=1$.

Now suppose the statement holds for $k$ and consider the set,

$$
\cup_{i=0}^{k+1} f^{-i p}\left(U_{r}\right)=f^{-(k+1) p}\left(U_{r}\right) \cup\left(\cup_{i=0}^{k} f^{-i p}\left(U_{r}\right)\right)=: f^{-(k+1) p}\left(U_{r}\right) \cup A_{k} .
$$

We claim that any intersection between $f^{-(k+1) p}\left(U_{r}\right)$ and $A_{k}$ necessarily belongs to $f^{-k p}\left(U_{r}\right)$. To see this, suppose $x \in f^{-(k+1) p}\left(U_{r}\right) \cap f^{-j p}\left(U_{r}\right)$ for some $j \leqslant k$. Then $f^{j p}(x) \in U_{r} \cap f^{-(k+1-j) p}\left(U_{r}\right)$, which necessarily remains in $U_{r}$ for the next $k+1-j$ iterates of $f^{p}$, due to the nested property of the sets $U_{r} \cap f^{-i p}\left(U_{r}\right)$. In particular, $f^{(k-j) p}\left(f^{j p} x\right) \in V_{r}$. Thus $x \in f^{-k p}\left(U_{r}\right)$. 
Using this fact about intersection as well as (2.7), we now estimate,

$$
\begin{aligned}
\mu\left(\cup_{i=0}^{k+1} f^{-i p}\left(U_{r}\right)\right) & =\mu\left(f^{-(k+1) p}\left(U_{r}\right)\right)+\mu\left(A_{k}\right)-\mu\left(f^{-(k+1) p}\left(U_{r}\right) \cap f^{-k p}\left(U_{r}\right)\right) \\
& =\mu\left(U_{r}\right)+\mu\left(A_{k}\right)-\mu\left(U_{r} \cap f^{-p}\left(U_{r}\right)\right) \\
& =\mu\left(U_{r}\right)+\mu\left(A_{k}\right)-(1 \pm \bar{C} \varepsilon) \mu\left(U_{r}\right) e^{S_{p} \varphi(z)},
\end{aligned}
$$

and the lemma is proved using the inductive hypothesis on $\mu\left(A_{k}\right)$.

Using the lemma, we may estimate

$$
\begin{gathered}
\frac{1}{\mu\left(U_{r}\right)} \log \mu\left(\tau_{r}>t\right)=\frac{1}{\mu\left(U_{r}\right)} \log \left(1-p \mu\left(\cup_{i=0}^{k} f^{-i p}\left(U_{r}\right)\right)\right) \\
=\frac{1}{\mu\left(U_{r}\right)} \log \left(1-\mu\left(U_{r}\right) p\left[k+1-k(1 \pm \varepsilon) e^{S_{p} \varphi(z)}\right]\right) \\
\underset{r \rightarrow 0}{\longrightarrow}-\left[p k+p-p k(1 \pm \bar{C} \varepsilon) e^{S_{p} \varphi(z)}\right] .
\end{gathered}
$$

Now dividing by $-t$ and taking the limit as $t \rightarrow \infty$ completes the proof of the periodic case, up to an error $\pm \varepsilon e^{S_{p} \varphi(z)}$. Since $\varepsilon$ was arbitrary, the case is proved for $t$ of the form $(k+1) p-1$.

For more general $t=k p+\ell$, for some $\ell=0, \ldots, p-1$, we have

$$
\mu\left(\tau_{r}>(k+1) p-1\right) \leqslant \mu\left(\tau_{r}>t\right) \leqslant \mu\left(\tau_{r}>k p-1\right),
$$

and since the upper and lower bounds yield the same limit as $k \rightarrow \infty$, the limit for general $t$ exists and has the same value.

2.5. Proof of Theorem 2.1: The case $\alpha \in(0, \infty)$. Fix $z \in I, \alpha \in(0, \infty)$, and a sequence of intervals $\left(U_{r}\right)_{r \in r_{0}}$ satisfying $(\mathbf{U})$. If $z$ is periodic, we also assume $(\mathbf{P})$. Let $t=s \mu_{0}\left(U_{r}\right)^{-\alpha}$ for some $s \in \mathbb{R}^{+}$. We must consider the limit,

$$
\lim _{r \rightarrow 0} \frac{1}{s \mu_{0}\left(U_{r}\right)^{1-\alpha}} \log \mu_{0}\left(\tau_{r}>s \mu_{0}\left(U_{r}\right)^{-\alpha}\right) .
$$

As in Section 2.1, there exists $r_{1}>0$ such that all associated transfer operators $\stackrel{\mathcal{L}}{r}_{r}$ have a uniform spectral gap on $\mathcal{B}$ for all $r \in\left[0, r_{1}\right]$.

To simplify notation, set $k_{r}=\left\lfloor s \mu_{0}\left(U_{r}\right)^{-\alpha}\right\rfloor$. Notice that,

$$
\begin{aligned}
\mu_{0}\left(\tau_{r}>k_{r}\right) & =\int_{\dot{I}_{r}^{k_{r}}} g_{0} d m_{0}=\int \dot{\mathcal{L}}_{r}^{k_{r}+1} g_{0} d m_{0} \\
& =\lambda_{r}^{k_{r}+1} \int \lambda_{r}^{-k_{r}-1} \dot{\mathcal{L}}_{r}^{k_{r}+1}\left(g_{0}-g_{r}\right) d m_{0}+\lambda_{r}^{k_{r}+1} \int g_{r} d m_{0},
\end{aligned}
$$

where $g_{r}$ is the unique normalised conditionally invariant density corresponding to $\lambda_{r}$ from Corollary 2.7. Thus

$$
\log \mu_{0}\left(\tau_{r}>k_{r}\right)=\left(k_{r}+1\right) \log \lambda_{r}+\log \left(1+\int \lambda_{r}^{-k_{r}-1}{\stackrel{\circ}{\mathcal{L}_{r}}}_{r}^{k_{r}+1}\left(g_{0}-g_{r}\right) d m_{0}\right) .
$$

Notice that the first term above, when divided by $s \mu_{0}\left(U_{r}\right)^{1-\alpha}$, is simply $\mu_{0}\left(U_{r}\right)^{-1} \log \lambda_{r}$ (up to integer part) and thus converges as $r \rightarrow 0$ to the required limit by (2.5), which depends on $z$. 
It remains to show that the second term in 2.8 converges to zero after division by $\mu_{0}\left(U_{r}\right)^{1-\alpha}$. Using Corollary 2.7, we may decompose the transfer operator as $\dot{\mathcal{L}}_{r}=\lambda_{r} \Pi_{r}+R_{r}$, where as before, $\Pi_{r}$ is the projection onto the eigenspace spanned by $g_{r}$ and the spectral radius of $R_{r}$ is strictly less than $\lambda_{r}$. Thus defining $\Pi_{r} g_{0}=c_{r} g_{r}$ for some $c_{r}>0$, we have

$$
\lambda_{r}^{-k_{r}-1} \mathcal{L}_{r}^{k_{r}+1}\left(g_{0}-g_{r}\right)=\left(c_{r}-1\right) g_{r}+\lambda_{r}^{-k_{r}-1} R_{r}^{k_{r}+1} g_{0},
$$

where we have used the facts, $\Pi_{r}^{2}=\Pi_{r}, \Pi_{r} g_{r}=g_{r}$ and $R_{r} g_{r}=0$. Integrating, we have

$$
\log \left(1+\int \lambda_{r}^{-k_{r}-1} \dot{\mathcal{L}}_{r}^{k_{r}+1}\left(g_{0}-g_{r}\right) d m_{0}\right)=\log \left(c_{r}+\int \lambda_{r}^{-k_{r}-1} R_{r}^{k_{r}+1} g_{0} d m_{0}\right) .
$$

Now since the operators $\dot{\mathcal{L}}_{r}$ have a uniform spectral gap for $r$ close to 0 , there exists $\beta>0$ such that the spectral radius of $\lambda_{r}^{-1} R_{r}$ in $\mathcal{B}$ is less than $e^{-\beta}$ for all $r$ sufficiently small. Since the variation norm dominates the $L^{\infty}$ norm, we have,

$$
\left|\int \lambda_{r}^{-k_{r}-1} R_{r}^{k_{r}+1} g_{0} d m_{0}\right| \leqslant\left\|\lambda_{r}^{-k_{r}-1} R_{r}^{k_{r}+1} g_{0}\right\| \leqslant C e^{-\beta\left(k_{r}+1\right)} \leqslant C e^{-\beta s \mu_{0}\left(U_{r}\right)^{-\alpha}},
$$

for some fixed $C>0$, and this quantity is super-exponentially small in $\mu_{0}\left(U_{r}\right)$. Moreover, since by [KL1, Corollary 1], the spectral projectors $\Pi_{r}$ of $\dot{\mathcal{L}}_{r}$ vary by at most $-\mu_{0}\left(U_{r}\right) \log \mu_{0}\left(U_{r}\right)$ for small $r$ and $\Pi_{0} g_{0}=g_{0}$, i.e., $c_{0}=1$, we have $\left|1-c_{r}\right| \leqslant-C \mu_{0}\left(U_{r}\right) \log \mu_{0}\left(U_{r}\right)$, for some uniform $C>0$.

Using these estimates in the second term of (2.8) and dividing by $s \mu_{0}\left(U_{r}\right)^{1-\alpha}$, the relevant expression becomes,

$$
\lim _{r \rightarrow 0} \frac{1}{s \mu_{0}\left(U_{r}\right)^{1-\alpha}} \log \left(1+\mathcal{O}\left(-\mu_{0}\left(U_{r}\right) \log \mu_{0}\left(U_{r}\right)\right)\right) .
$$

For $\alpha \geqslant 1$, it suffices to note that $\log \left(1+\mathcal{O}\left(-\mu_{0}\left(U_{r}\right) \log \mu_{0}\left(U_{r}\right)\right)\right)$ converges to 0 as $r \rightarrow 0$ to conclude that the above limit vanishes. For $\alpha \in(0,1)$, we note that in addition $\frac{\mu_{0}\left(U_{r}\right) \log \mu_{0}\left(U_{r}\right)}{\mu_{0}\left(U_{r}\right)^{1-\alpha}} \rightarrow$ 0 as $r \rightarrow 0$, which completes the proof of Theorem 2.1.

2.6. Examples. In this section, we provide examples of several classes of maps and potentials for which our assumptions (F1)-(F4) of Section 2 hold. More general examples, including the existence of a conformal measure for contracting potentials, can be constructed using [LSV1].

2.6.1. Lasota-Yorke maps of the interval with $\varphi=-\log |D f|$. Such maps are assumed to admit a finite partition $\mathcal{Z}$ of $I$ into intervals on which $f$ is differentiable and $|D f| \geqslant \sigma^{-1}>1$. $f$ is assumed to be $C^{2}$ on the closure of each $Z \in \mathcal{Z}$.

The conformal measure $m$ is Lebesgue measure on $I$, and (F1)-(F3) are standard consequences of uniform expansion, the existence of $D^{2} f$ and the finiteness of the partition $\mathcal{Z}$.

Since the potential is bounded, condition (F4) can be guaranteed by the equivalent condition that for each interval $J$, there exists $n(J)$, such that $f^{n(J)}(J)=I \bmod 0$.

Once we fix $z=\cap_{r>0} U_{r}$ and $n_{1}$ from (2.1), (U) is always satisfied for $r$ sufficiently small due to the finiteness of $\mathcal{Z}^{n_{1}}$. Thus Theorem 2.1 holds for this class of maps. 
2.6.2. Mixing Gibbs-Markov maps with large images. Assume that $f(Z)$ is a union of elements of $\mathcal{Z}$ for each $Z \in \mathcal{Z}$, where $\mathcal{Z}$ is the countable partition defined at the beginning of Section 2 . Thus $\mathcal{Z}$ is a Markov partition for $f$.

We assume that $f$ satisfies the big images and pre-images ${ }^{3}(\mathrm{BIP})$ property: there exists a finite set $\left\{Z_{j}\right\}_{j \in \mathcal{J}} \subset \mathcal{Z}$ such that $\forall Z \in \mathcal{Z}, \exists j, k \in \mathcal{J}$ such that $f\left(Z_{j}\right) \supseteq Z$ and $f(Z) \supseteq Z_{k}$. We also assume that $|D f| \geqslant \sigma^{-1}>1$ on each $Z \in \mathcal{Z}$.

We assume that $\varphi$ is a potential which is Lipschitz continuous on each $Z \in \mathcal{Z}$, and admits a non-atomic conformal probability measure $m_{\varphi}$ with $m_{\varphi}\left(I \backslash \cup_{Z \in \mathcal{Z}} Z\right)=0$.

Then (F1) follows immediately from the regularity of $\varphi$ and the expansion of $f$, and we have a Gibbs-Markov map. Condition (F2) follows from the existence of $m_{\varphi}$ and (BIP) since by (F1), $\sup _{Z} e^{\varphi} \leqslant\left(1+C_{d}\right) m_{\varphi}(Z) / m_{\varphi}(f(Z))$ :

$$
\sum_{Z \in \mathcal{Z}} \sup _{Z} e^{\varphi} \leq\left(1+C_{d}\right) \sum_{Z \in \mathcal{Z}} \frac{m_{\varphi}(Z)}{m_{\varphi}(f(Z))} \leqslant\left(1+C_{d}\right) c_{0}^{-1}
$$

where $c_{0}=\inf _{Z \in \mathcal{Z}} m_{\varphi}(f(Z))>0$ by (BIP).

(F4) follows from mixing plus (BIP). For (F3), we use the fact that for maps satisfying our assumptions, the transfer operator $\mathcal{L}_{\varphi}$ acting on functions which are Lipschitz on each element of $\mathcal{Z}$ is known to have a spectral gap. Since $\mathcal{L}_{\varphi}^{n} 1$ converges to an invariant density that is bounded away from 0 (by (F4)), the expression on the right side of (F3) is bounded away from 0 for all $n$ large enough. On the other hand, the expression on the left side of (F3) must tend to 0 by conformality and (F1), since for $Z \in \mathcal{Z}^{n}$,

$$
\sup _{Z} e^{S_{n} \varphi} \leqslant\left(1+C_{d}\right) \frac{m_{\varphi}(Z)}{m_{\varphi}\left(f^{n} Z\right)} \leqslant\left(1+C_{d}\right) \frac{m(Z)}{c_{0}},
$$

and the diameter of $\mathcal{Z}^{n}$ must tend to 0 by the expansivity of $f$.

Having verified (F1)-(F4), we may apply Theorem 2.1 to this class of Gibbs-Markov maps. Note that we can always arrange for $(\mathbf{U})$ to be satisfied as long as we do not choose $\cap_{r} U_{r}$ to be an accumulation point of the endpoints of the intervals in $\mathcal{Z}^{n_{1}}$.

2.6.3. Gauss map, $f(x)=1 / x \bmod 1$. In this case, $\varphi=-\log |D f|, m_{\varphi}$ is Lebesgue measure on $[0,1]$, the invariant density is $g_{0}=\frac{1}{\ln 2} \frac{1}{1+x}$, and $f$ is continuously differentiable on each element of the partition $\mathcal{Z}=\left\{Z_{j}\right\}_{j=1}^{\infty}, Z_{j}=(1 /(j+1), 1 / j)$.

For this potential, (F1) fails. However this system is well known to satisfy Rychlik's conditions since the potential is monotonic on each branch; moreover the potential satisfies the weaker (Hölder) distortion control given by the following lemma.

Lemma 2.9. $\exists C_{d}>0$ s.t. $\left|e^{S_{n} \varphi(x)-S_{n} \varphi(y)}-1\right| \leqslant C_{d}\left|f^{n} x-f^{n} y\right|^{1 / 2}$, whenever $f^{i} x$, $f^{i} y$ lie in the same element of $\mathcal{Z}$ for $i=0,1, \ldots, n-1$.

Before proving the lemma, we will verify the other conditions and show that in this case, Lemma 2.9 suffices to prove Proposition 2.5, so that the conclusions of Theorem 2.1 hold.

\footnotetext{
${ }^{3}$ This is automatic if $f$ is full-branched.
} 
(F2) is immediate since $\sup _{Z_{j}} e^{\varphi} \leqslant C j^{-2}, \forall j \geqslant 1$.

Notice that $\left|e^{\varphi}\right|_{\infty} \leqslant 1$, while $\left|e^{S_{2} \varphi}\right|_{\infty}<1$. Thus the expression on the left side of (F3) decreases exponentially in $n$, while $\mathcal{L}_{\varphi}^{n} 1$ converges to $g_{0}$, which is bounded away from 0 on $I$. Thus (F3) holds.

(F4) holds since $f$ is full-branched, and the potential satisfies the distortion control given by Lemma 2.9.

We verify also that the items of Lemma 2.4 hold: (a) holds by induction on (F2); (b) holds with $C_{d}=1$ since $e^{\varphi}$ is monotonic on each $Z_{j}$, so that $\bigvee_{Z_{j}} e^{\varphi} \leqslant \sup _{Z_{j}} e^{\varphi} ;$ (c) holds by induction on (b), using (a).

Next we show that the operators $\stackrel{\circ}{\mathcal{L}}_{r}$ satisfy the uniform Lasota-Yorke inequalities of Proposition 2.5 under assumption (U). The assumption (F1) is used in precisely two places in the proof of the proposition: in equations A.2 and A.3 . For A.2), the Hölder distortion control given by Lemma 2.9 suffices to give precisely the same bound. For $(\mathrm{A} .3)$, we use $\bigvee_{J_{i}} e^{S_{n} \varphi} \leqslant \sup _{J_{i}} e^{S_{n} \varphi}$ by the monotonicity of $e^{S_{n} \varphi}$ on each $J_{i}$.

With these estimates, the contracting term in (A.4) becomes $4\left|e^{S_{n} \varphi}\right|_{\infty} \bigvee_{I} \psi$ (it is the same expression, but with $\left.C_{d}=1\right)$. Thus we need only choose $n_{1}$ such that $4\left|e^{S_{n_{1}} \varphi}\right|_{\infty}<1$, replacing (2.1), in order to prove the required Lasota-Yorke inequalities under assumption (U). Note that since $f$ is full-branched, we can arrange for (U) to be satisfied as long as $\{z\}=\cap_{r} U_{r}$ is not chosen to be an endpoint of $\mathcal{Z}^{n_{1}}$.

Turning to the proof of Theorem 2.1, condition (F1) is used directly in one additional place: the proof of Lemma 2.8. In that case, using the Hölder bound given by Lemma 2.9, we need only replace $\operatorname{diam}\left(U_{r}\right)$ by $\sqrt{\operatorname{diam}\left(U_{r}\right)}$ and choose $U_{r}$ sufficiently small that $\sqrt{\operatorname{diam}\left(U_{r}\right)}<\varepsilon$. Then the rest of the proof of Lemma 2.6 goes through without changes.

With these minor changes to the proof, the conclusions of Theorem 2.1 apply to the Gauss map.

Proof of Lemma 2.9. Let $x, y$ be as in the statement of the lemma, and let $f^{i} x \in Z_{j_{i}}$. The following bounds are elementary, yet essential to what follows,

$$
\sup _{Z_{j}} \frac{\left|D f^{2}\right|}{|D f|} \leqslant C j \quad \text { while } \quad \operatorname{diam}\left(Z_{j}\right) \leqslant C j^{-2} .
$$

Using these estimates, one may complete the standard (Hölder) distortion estimate,

$$
\begin{aligned}
\left|\log \frac{D f^{n}(x)}{D f^{n}(y)}\right| & \leqslant \sum_{i=0}^{n-1}|\log | D f\left(f^{i} x\right)|-\log | D f\left(f^{i} y\right)|| \\
& \leqslant \sum_{i=0}^{n-1} \sup _{Z_{j_{i}}} \frac{\left|D f^{2}\right|}{|D f|}\left|f^{i} x-f^{i} y\right| \leqslant \sum_{i=0}^{n-1} C\left|f^{i} x-f^{i} y\right|^{1 / 2} \\
& \leqslant C\left|f^{n} x-f^{n} y\right|^{1 / 2} \sum_{i=0}^{n-1}\left|e^{S_{n-i} \varphi}\right|_{\infty}^{1 / 2} .
\end{aligned}
$$


The final sum converges exponentially in $i$ because $\left|e^{\varphi}\right|_{\infty} \leqslant 1$ and $\left|e^{S_{2} \varphi}\right|_{\infty}<1$.

\section{Results VIA INDUCinG}

In this section, we consider some cases in which the map $f: I \rightarrow I$ and potential $\varphi$ do not satisfy (F1)-(F4) of Section 2. In such cases, a common strategy is to consider an induced map to a subset of $I$ with stronger statistical properties. This is the situation we shall address in this section: explicit examples will be given in the following section.

We begin with a map $f: I \rightarrow I$, a conformal measure $m_{\varphi}$ with potential $\varphi$, and an invariant probability measure $\mu_{\varphi}$, absolutely continuous with respect to $m_{\varphi}$. We will fix the potential and simply denote this measure by $\mu$ in this section.

Fixing a sequence of sets $\left(U_{r}\right)_{r \in\left[0, r_{0}\right]}$, we assume that we can select an interval $Y$ with $\mu(Y)>0$ and $U_{r} \subset Y \subset I$, such that the first return map $F=f^{R_{Y}}: Y \rightarrow Y$ and the induced potential $\Phi=\sum_{i=0}^{R_{Y}-1} \varphi \circ f^{i}$ satisfy (F1)-(F4).

Let $\mu_{Y}:=\left.\frac{1}{\mu(Y)} \mu\right|_{Y}$ be the $F$-invariant probability measure, and $\tau_{Y, r}(y)=\min \left\{u \geqslant 1: F^{u}(y) \in\right.$ $U_{r}$ \} be the first hitting time for the set $U_{r}$, which we sometimes refer to as the hole. Let $R_{Y, u}(y)=\sum_{i=0}^{u-1} R_{Y} \circ F^{i}(y)$ be the $u^{\text {th }}$ return time to $Y$.

Remark 3.1. We will assume for simplicity that the hole is always in $Y$. This is not much of a restriction because it is generically possible, once the location of the hole is known (and it is not at an indifferent fixed point or a recurrent critical point), to select a set $Y$ with good return map containing the hole.

For $\mu_{Y}$-a.e. $y \in Y$, we have $R_{Y, u} / u \rightarrow 1 / \mu(Y)$, but for our purposes we need specific estimates for the large deviations $\mu_{Y}\left(A_{u}\right)$ for the set

$$
A_{u}=A_{Y, u, \varepsilon}:=\left\{y \in Y: \exists n \geqslant u \text { such that }\left|R_{Y, n}-n / \mu(Y)\right|>n \varepsilon\right\} .
$$

Following (2.2), we define $Y_{\text {cont }}$ to be the set of points in $Y$ at which $F^{k}$ is continuous for all $k \in \mathbb{N}$.

Theorem 3.2. Suppose $f: I \rightarrow I$ is as above and there exists $Y \subset I$ with $z \in Y_{\text {cont }}$ such that the first return map $F=f^{R_{Y}}: Y \rightarrow Y$ satisfies the assumptions of Theorem 2.1.

(1) If for any small $\varepsilon>0$, there exists $c(\varepsilon)>0$ such that $\mu_{Y}\left(A_{u}\right) \leqslant e^{-c(\varepsilon) u}$ for all large $u$, then for each $\alpha \in(0, \infty]$,

$$
L_{\alpha, s}(z)= \begin{cases}1, & \text { if } z \text { is not periodic, } \\ 1-e^{S_{p} \varphi(z)}, & \text { if } z \text { is } p \text {-periodic for } f .\end{cases}
$$

(2) If there exist $\gamma \in(0,1)$ such that for any small $\varepsilon>0$, there exist $C, c(\varepsilon)>0$ such that $\mu_{Y}\left(A_{u}\right) \leqslant C e^{-c(\varepsilon) u^{\gamma}}$ for all large $u$, then (3.1) holds for each $\alpha<\frac{1}{1-\gamma}$.

(3) If there exists $\gamma \in(0,1)$ and $C, c>0$ such that $\mu_{Y}\left(R_{Y} \geqslant u\right) \geqslant C e^{-c u^{\gamma}}$ for all large $u$, then $L_{\alpha, s}(z)=0$ for $\alpha>\frac{1}{1-\gamma}$ and each $z \in I$. 
(4) If both $\mu_{Y}\left(A_{u}\right)$ and $\mu_{Y}\left(R_{Y} \geqslant u\right)$ decay superpolynomially in $u$, but more slowly than any stretched exponential, then (3.1) holds if $\alpha \leqslant 1$ and $L_{\alpha, s}(z)=0$ if $\alpha>1$ for each $z \in I_{\text {cont }}$.

Remark 3.3. One expects, as in the examples of Section 4, that the decay of $\mu_{Y}\left(R_{Y} \geqslant u\right)$ matches that of $\mu_{Y}\left(A_{u}\right)$, so (2) and (3) in this theorem can be seen as complementary cases.

Remark 3.4. Theorem 3.2 excludes the case $\alpha=0$ since as already noted in Remark 2.3, the limit holds in this case under general conditions which do not require a spectral gap. Thus it is not necessary to pass to the induced map $F$ in this case; one simply needs to verify the conditions listed in Remark 2.3.

3.1. Proof of Theorem 3.2. We first prove the theorem for the quantity

$$
L_{Y, \alpha, s}(z):=\lim _{r \rightarrow 0} \frac{-1}{s \mu\left(U_{r}\right)^{1-\alpha}} \log \mu_{Y}\left(\tau_{r}>s \mu\left(U_{r}\right)^{-\alpha}\right) .
$$

Proposition 3.5. Under the conditions of Theorem 3.2, all parts of the theorem hold with $L_{Y, \alpha, s}(z)$ replacing $L_{\alpha, s}(z)$. In particular, in cases (1) and (2),

$$
L_{Y, \alpha, s}(z)= \begin{cases}1, & \text { if } z \text { is not periodic, } \\ 1-e^{S_{p} \varphi(z)}, & \text { if } z \text { is p-periodic for } f\end{cases}
$$

Proof. We will assume throughout that $\alpha \neq 1$ since this case is straightforward and proved elsewhere. Fix some small $\varepsilon>0$, and assume that the hole $U_{r}$ is contained inside one domain of $F$. For notational simplicity, here we will assume that the centre $z$ of our $U_{r}$ is non-periodic, but the periodic case is then immediate. We remark only that if $z$ is periodic for $f$ with period $p$ and in the domain of $F$, then $z$ is periodic for $F$ with period $q \leqslant p$, and $e^{S_{q} \Phi(z)}=e^{S_{p} \varphi(z)}$.

If $y \in A_{u}^{c}$ and $t=u / \mu(Y)$, then $\tau_{r}(y)>t$ implies that $\tau_{Y, r}(y)>u /(1+\varepsilon \mu(Y))$ and is implied by $\tau_{Y, r}(y)>u /(1-\varepsilon \mu(Y))$. Since Theorem 2.1 applies to $\left(F, Y, \mu_{Y}\right)$, there exist values $\theta^{+}(v, r), \theta^{-}(v, r)$ so that

$$
\theta^{-}(v, r) e^{-v \mu_{Y}\left(U_{r}\right)^{1-\alpha}} \leqslant \mu_{Y}\left(\tau_{Y, r}>v \mu_{Y}\left(U_{r}\right)^{-\alpha}\right) \leqslant \theta^{+}(v, r) e^{-v \mu_{Y}\left(U_{r}\right)^{1-\alpha}},
$$

where $\lim _{r \rightarrow 0} \frac{\log \theta^{ \pm}(v, r)}{v \mu\left(U_{r}\right)^{1-\alpha}}=0$.

We compute for the path $t=s \mu\left(U_{r}\right)^{-\alpha}$, so

$$
u=s \mu(Y) \mu\left(U_{r}\right)^{-\alpha}=s \mu(Y)^{1-\alpha} \mu_{Y}\left(U_{r}\right)^{-\alpha} .
$$

We write $\theta^{-}(r)=\theta^{-}\left(s(1-\varepsilon \mu(Y))^{-1} \mu(Y)^{1-\alpha}, r\right)$ and $\theta^{+}(r)=\theta^{+}\left(s(1+\varepsilon \mu(Y))^{-1} \mu(Y)^{1-\alpha}, r\right)$ to shorten notation. Also we abbreviate

$$
G^{ \pm}=\left\{y \in Y: \tau_{Y, r}(y)>s(1 \pm \varepsilon \mu(Y))^{-1} \mu(Y)^{1-\alpha} \mu_{Y}\left(U_{r}\right)^{-\alpha}\right\} .
$$


First we bound $\mu_{Y}\left(\tau_{r}>t\right)$ from above, since $\left\{\tau_{r}>t \wedge A_{u}^{c}\right\} \subset G^{+}$,

$$
\begin{aligned}
\mu_{Y}\left(\tau_{r}>t\right) & =\mu_{Y}\left(\tau_{r}>t \wedge A_{u}^{c}\right)+\mu_{Y}\left(\tau_{r}>t \wedge A_{u}\right) \\
& \leqslant \mu_{Y}\left(G^{+}\right)+\mu_{Y}\left(A_{u}\right) \\
& \leqslant \theta^{+}(r) e^{-s(1+\varepsilon \mu(Y))^{-1} \mu(Y)^{1-\alpha} \mu_{Y}\left(U_{r}\right)^{1-\alpha}}+\mu_{Y}\left(A_{u}\right) \\
& =\theta^{+}(r) e^{-s(1+\varepsilon \mu(Y))^{-1} \mu\left(U_{r}\right)^{1-\alpha}}+\mu_{Y}\left(A_{u}\right) .
\end{aligned}
$$

Similarly, we bound $\mu_{Y}\left(\tau_{r}>t\right)$ from below, using $G^{-}$:

$$
\begin{aligned}
\mu_{Y}\left(\tau_{r}>t \wedge A_{u}^{c}\right) & \geqslant \mu_{Y}\left(\tau_{Y, r}>u(1-\varepsilon \mu(Y))^{-1} \wedge A_{u}^{c}\right) \\
& \geqslant \mu_{Y}\left(\tau_{Y, r}>s(1-\varepsilon \mu(Y))^{-1} \mu(Y) \mu\left(U_{r}\right)^{-\alpha}\right)-\mu_{Y}\left(G^{-} \wedge A_{u}\right) \\
& \geqslant \theta^{-}(r) e^{-s(1-\varepsilon \mu(Y))^{-1} \mu\left(U_{r}\right)^{1-\alpha}}-\mu_{Y}\left(G^{-} \wedge A_{u}\right),
\end{aligned}
$$

and therefore,

$$
\begin{aligned}
\mu_{Y}\left(\tau_{r}>t\right) & =\mu_{Y}\left(\tau_{r}>t \wedge A_{u}^{c}\right)+\mu_{Y}\left(\tau_{r}>t \wedge A_{u}\right) \\
& \geqslant \theta^{-}(r) e^{-s(1-\varepsilon \mu(Y))^{-1} \mu\left(U_{r}\right)^{1-\alpha}}+\mu_{Y}\left(\tau_{r}>t \wedge A_{u}\right)-\mu_{Y}\left(G^{-} \wedge A_{u}\right) \\
& \geqslant \theta^{-}(r) e^{-s(1-\varepsilon \mu(Y))^{-1} \mu\left(U_{r}\right)^{1-\alpha}}-\mu_{Y}\left(A_{u}\right) .
\end{aligned}
$$

Now to find the limit in $(3.2)$, we use first $(3.3)$ to bound $L_{Y, \alpha, s}$ from below (taking a minus sign, so the inequality flips):

$$
\begin{aligned}
& \frac{-\log \mu_{Y}\left(\tau_{r}>t\right)}{s \mu\left(U_{r}\right)^{1-\alpha}} \geqslant-\frac{\log \left(\theta^{+}(r) e^{-s(1+\varepsilon \mu(Y))^{-1} \mu\left(U_{r}\right)^{1-\alpha}}+\mu_{Y}\left(A_{u}\right)\right)}{s \mu\left(U_{r}\right)^{1-\alpha}} \\
& =-\frac{\log \theta^{+}(r)}{s \mu\left(U_{r}\right)^{1-\alpha}}+\frac{1}{1+\varepsilon \mu(Y)}-\frac{\log \left(1+\frac{e^{s(1+\varepsilon \mu(Y))^{-1} \mu\left(U_{r}\right)^{1-\alpha}}}{\theta^{+}(r)} \mu_{Y}\left(A_{u}\right)\right)}{s \mu\left(U_{r}\right)^{1-\alpha}} .
\end{aligned}
$$

The first term converges to zero as $r \rightarrow 0$ by assumption, so we focus on the final term.

Case I: $\alpha \in(\mathbf{0}, \mathbf{1})$. In this case since $\theta^{+}(r)=O\left(e^{s \mu\left(U_{r}\right)^{1-\alpha}+\delta}\right)$ for any $\delta>0$, and $\mu\left(U_{r}\right)^{1-\alpha} \rightarrow 0$ as $r \rightarrow 0$, hence $\frac{e^{-s(1+\varepsilon \mu(Y))^{-1} \mu\left(U_{r}\right)^{1-\alpha}}}{\theta^{+}(r)}=O(1)$ and we see that the final term of $(3.5)$ is of order $\frac{\mu_{Y}\left(A_{u}\right)}{\mu\left(U_{r}\right)^{1-\alpha}}$. Assuming that $\mu_{Y}\left(A_{u}\right) \leqslant C u^{-\beta}$ for some $C, \beta>0$, we have,

$$
\mu_{Y}\left(A_{u}\right) \leqslant C\left(s \mu(Y) \mu\left(U_{r}\right)^{-\alpha}\right)^{-\beta},
$$

so that $L_{Y, \alpha, s}(z) \geqslant 1 /(1+\varepsilon \mu(Y))$ if $\alpha-1+\alpha \beta>0$, i.e., $\alpha>\frac{1}{1+\beta}$. So the lower bound for the non-degenerate part (i.e., the " $\alpha \leq 1$ " part) of (4) follows along with (2) and (1) for the $\alpha \in(0,1)$ case since $\varepsilon$ was arbitrary. The upper bound follows immediately from Remark 1.1 .

Case II: $\alpha \in(\mathbf{1}, \infty)$. Here we focus on the stretched exponential case since all remaining parts of this proposition then follow. To complete the proof of (2), we again refer to (3.5). Suppose that there exist $C, c(\varepsilon)>0$ and $\gamma \in(0,1)$ such that $\mu_{Y}\left(A_{u}\right) \leqslant C e^{-c(\varepsilon) u^{\gamma}}$. Then for (3.2) to hold it is sufficient that the decay of $\mu_{Y}\left(A_{u}\right)$, which is $C e^{-c\left(s \mu\left(U_{r}\right)^{-\alpha}\right)^{\gamma}}$, is faster than 
$e^{-s(1+\varepsilon \mu(Y))^{-1} \mu\left(U_{r}\right)^{1-\alpha}}$. So we require that $\alpha<\frac{1}{1-\gamma}$. The upper bound follows similarly, using (3.4) in place of (3.3), completing (2).

To prove (3) and the degenerate (i.e., " $\alpha>1$ ") part of (4), we assume that there exist $C, c>0$, $\gamma \in(0,1)$ such that $\mu_{Y}\left(R_{Y} \geqslant t\right) \geqslant C e^{-c t^{\gamma}}$. Fix $\alpha>\frac{1}{1-\gamma}$. Then using the fact that $\left\{\tau_{r}>t\right\} \supset$ $\left\{R_{Y} \geqslant t\right\}$, we estimate

$$
\begin{aligned}
\frac{-\log \mu_{Y}\left(\tau_{r}>t\right)}{s \mu\left(U_{r}\right)^{1-\alpha}} & \leqslant \frac{-\log \mu_{Y}\left(R_{Y} \geqslant t\right)}{s \mu\left(U_{r}\right)^{1-\alpha}} \leqslant \frac{-\log \left(C e^{-c t^{\gamma}}\right)}{s \mu\left(U_{r}\right)^{1-\alpha}} \\
& \leqslant \frac{-\log C}{s \mu\left(U_{r}\right)^{1-\alpha}}+\frac{c s^{\gamma} \mu\left(U_{r}\right)^{-\alpha \gamma}}{s \mu\left(U_{r}\right)^{1-\alpha}}
\end{aligned}
$$

and both terms tend to 0 with $r$ since $\alpha>1 /(1-\gamma)$. Note that this estimate easily extends from the measure $\mu_{Y}$ to the measure $\mu$, so that $L_{\alpha, s}(z)=0$ for all $z \in I$.

Case III: $\alpha=\infty$. For this case, we compute first the limit $t \rightarrow \infty$ and then $r \rightarrow 0$ in the expression given by 1.4 .

Fix $\varepsilon>0$ and define $A_{u}$ as before. In analogy to the previous two cases, set

$$
G^{ \pm}=\left\{y \in Y: \tau_{Y, r}(y)>u /(1 \pm \varepsilon \mu(Y))\right\}
$$

where $u=t \mu(Y)$. Notice then that $\left\{\tau_{r}>t \wedge A_{u}^{c}\right\} \subset G^{+}$as before. Thus as in (3.3),

$$
\mu_{Y}\left(\tau_{r}>t\right) \leqslant \mu_{Y}\left(G^{+}\right)+\mu_{Y}\left(A_{u}\right) .
$$

Following (3.4), we obtain,

$$
\mu_{Y}\left(\tau_{r}>t\right) \geqslant \mu_{Y}\left(G^{-}\right)-\mu_{Y}\left(A_{u}\right)
$$

To prove the exponential case (1), assume that there exists $c(\varepsilon)>0$ such that $\mu_{Y}\left(A_{u}\right) \leqslant$ $C e^{-c(\varepsilon) u}$. Since $F$ satisfies the assumptions of Theorem 2.1, we only consider $r$ so small such that all associated transfer operators $\dot{\mathcal{L}}_{r}$ have a uniform spectral gap. Let $\Lambda_{r}$ denote the leading eigenvalue of $\dot{\mathcal{L}}_{r}$ and choose $r_{1}$ so small $\Lambda_{r}^{(1-\varepsilon \mu(Y))^{-1}}>e^{-c(\varepsilon)}$ for all $r<r_{1}$. By Corollary 2.7.

$$
C^{-1} \Lambda_{r}^{u(1 \pm \varepsilon \mu(Y))^{-1}} \leqslant \mu_{Y}\left(G^{ \pm}\right) \leqslant C \Lambda_{r}^{u(1 \pm \varepsilon \mu(Y))^{-1}},
$$

for some $C>0$, independent of $t$, but possibly depending on $r$.

Thus on the one hand we derive a lower bound,

$$
\begin{aligned}
\lim _{t \rightarrow \infty}-\frac{1}{t} \log \mu_{Y}\left(\tau_{r}>t\right) & \geqslant \lim _{t \rightarrow \infty}-\frac{1}{t} \log \left(C \Lambda_{r}^{t \mu(Y) /(1+\varepsilon \mu(Y))}+C e^{-c(\varepsilon) t \mu(Y)}\right) \\
& =\frac{-\mu(Y) \log \Lambda_{r}}{1+\varepsilon \mu(Y)} .
\end{aligned}
$$

On the other hand, the analogous upper bound holds,

$$
\begin{aligned}
\lim _{t \rightarrow \infty}-\frac{1}{t} \log \mu_{Y}\left(\tau_{r}>t\right) & \leqslant \lim _{t \rightarrow \infty}-\frac{1}{t} \log \left(C^{-1} \Lambda_{r}^{t \mu(Y) /(1-\varepsilon \mu(Y))}-C e^{-c(\varepsilon) t \mu(Y)}\right) \\
& =\frac{-\mu(Y) \log \Lambda_{r}}{1-\varepsilon \mu(Y)} .
\end{aligned}
$$


Since $\varepsilon>0$ was arbitrary, this yields

$$
\lim _{t \rightarrow \infty}-\frac{1}{t} \log \mu_{Y}\left(\tau_{r}>t\right)=\lim _{t \rightarrow \infty}-\frac{1}{t} \log \mu\left(\tau_{r}>t \wedge Y\right)=-\mu(Y) \log \Lambda_{r} .
$$

Now using (2.5) applied to the induced map $F$, we conclude

$$
\lim _{r \rightarrow 0} \lim _{t \rightarrow \infty}-\frac{1}{t \mu\left(U_{r}\right)} \log \mu_{Y}\left(\tau_{r}>t\right)=\lim _{r \rightarrow 0} \frac{-\log \Lambda_{r}}{\mu_{Y}\left(U_{r}\right)}=1
$$

in the generic case, and $1-e^{S_{p} \varphi(z)}$ in the periodic case.

For the remaining items (2)-(4) of the proposition, it suffices to show that $L_{Y, \alpha, s}(z)=0$ when $\alpha=\infty$ under the assumption that $\mu_{Y}\left(R_{Y} \geqslant t\right) \geqslant C e^{-c t^{\gamma}}$ for some $\gamma \in(0,1)$. This is a trivial estimate since in this case the escape rate is 0 , i.e.,

$$
0 \leqslant \lim _{t \rightarrow \infty}-\frac{1}{t} \log \mu_{Y}\left(\tau_{r}>t\right) \leqslant \lim _{t \rightarrow \infty}-\frac{1}{t} \log \mu_{Y}\left(R_{Y} \geqslant t\right) \leqslant \lim _{t \rightarrow \infty} c t^{\gamma-1}=0 .
$$

It follows immediately that $L_{Y, \alpha, s}(z)=0$ for all $z \in Y$.

Proof of Theorem 3.2. We will apply Proposition 3.5 to convert the results for $L_{Y, \alpha, s}$ to $L_{\alpha, s}$. For this, we turn to an extended system implied by the existence of the first return map $F$. We will refer to this as a Rokhlin tower (our map $F$ defines what is nearly a Young tower, see [Y], except that we do not require that $F$ have a Markov structure). Define

$$
\Delta=\left\{(y, n) \in Y \times \mathbb{N}: n<R_{Y}(y)\right\} .
$$

The $\ell$ th level of the tower is $\Delta_{\ell}=\{(y, n) \in \Delta: n=\ell\}$ and the dynamics is defined by $f_{\Delta}(y, n)=(y, n+1)$ if $n<R_{Y}(y)-1$ and $f_{\Delta}\left(y, R_{Y}(y)-1\right)=(F(y), 0)$. The first return map to the base of the tower $\Delta_{0}=Y$ is again $F=f^{R_{Y}}$.

The assumptions on $F$ imply that $\mu_{Y}$ is an invariant probability measure on $Y=\Delta_{0}$, which induces an $f_{\Delta}$-invariant probability measure $\mu_{\Delta}$ on $\Delta$ : Define $\left.\mu_{\Delta}\right|_{\Delta_{\ell}}=\left.c\left(f_{\Delta}\right)_{*}^{\ell} \mu_{Y}\right|_{f_{\Delta}^{-\ell}\left(\Delta_{\ell}\right)}$, where $c=\mu_{Y}\left(R_{Y}\right)^{-1}$ is the normalising constant. Letting $\pi: \Delta \rightarrow I$ denote the natural projection, $\pi(x, \ell)=f^{\ell}(x)$, we have $\pi \circ f_{\Delta}=f \circ \pi$ and $\pi_{*} \mu_{\Delta}=\mu$.

Letting $\Delta^{(n)}=\cup_{\ell=0}^{n} \Delta_{\ell}$ denote the $n$ first levels of the tower, we observe that this gives us a sequence of induced maps $F_{n}: \Delta^{(n)} \rightarrow \Delta^{(n)}$ each satisfying the conditions of Theorem 2.1. (In fact, using the assumption on $F$, the potential for $\left(F_{n}\right)^{n}$ is contracting.) The projection $\pi\left(\Delta^{(n)}\right)$ gives a sequence of sets exhausting the space: $\mu\left(\pi\left(\Delta^{(n)}\right)\right) \rightarrow 1$ as $n \rightarrow \infty$. We will carry out the proof for $\pi\left(\Delta^{(n)}\right)$ in place of $Y$, but calling it $Y$ again and suppressing the index $n$.

Case I: $\alpha \in(\mathbf{0}, \mathbf{1})$. We will use the facts

(a) $\mu\left(\tau_{r} \leqslant t \wedge Y\right) \leqslant \mu\left(\tau_{r} \leqslant t\right)$

(b) given $\gamma \in \mathbb{R}$, for $x$ small, $\log (1+\gamma x) \sim \gamma \log (1+x)$ 
Then for $\alpha \in(0,1), t=s \mu\left(U_{r}\right)^{-\alpha}$,

$$
\begin{aligned}
-\frac{\log \mu_{Y}\left(\tau_{r}>t\right)}{s \mu\left(U_{r}\right)^{1-\alpha}} & =-\frac{\log \left(1-\mu_{Y}\left(\tau_{r} \leqslant t\right)\right)}{s \mu\left(U_{r}\right)^{1-\alpha}}=-\frac{\log \left(1-\mu(Y)^{-1} \mu\left(\tau_{r} \leqslant t \wedge Y\right)\right)}{s \mu\left(U_{r}\right)^{1-\alpha}} \\
& \leqslant-\frac{\log \left(1-\mu(Y)^{-1} \mu\left(\tau_{r} \leqslant t\right)\right)}{s \mu\left(U_{r}\right)^{1-\alpha}} \sim-\frac{1}{\mu(Y)} \frac{\log \left(1-\mu\left(\tau_{r} \leqslant t\right)\right)}{s \mu\left(U_{r}\right)^{1-\alpha}} \\
& =-\frac{1}{\mu(Y)} \frac{\log \mu\left(\tau_{r}>t\right)}{s \mu\left(U_{r}\right)^{1-\alpha}} .
\end{aligned}
$$

Here we used (a) in the ' $\leqslant$ step' and (b) in the ' $\sim$ step'. So choosing $Y=\pi\left(\Delta^{(n)}\right)$ exhausting our phase space, we deduce

$$
\liminf _{r \rightarrow 0} \frac{-\log \mu\left(\tau_{r}>t\right)}{s \mu\left(U_{r}\right)^{1-\alpha}} \geqslant \begin{cases}1, & \text { if } z \text { is not periodic, } \\ 1-e^{S_{p} \varphi(z)}, & \text { if } z \text { is } p \text {-periodic for } f .\end{cases}
$$

For non-periodic $z$, Remark 1.1 gives the upper bound as 1 too, so $L_{\alpha, s}(z)=1$.

For the periodic case we adapt Remark 1.1 and use a result of [FFT2]. First recall $V_{r}:=$ $U_{r} \cap f^{-p}\left(U_{r}\right)$ from the proof of Lemma 2.8 and let $V_{r}^{\prime}:=U_{r} \backslash f^{-p}\left(U_{r}\right)$. For all small $r$, this will be a topological annulus around $z$. By conditions (P) and (F1) (see (2.7)),

$$
\lim _{r \rightarrow 0} \frac{\mu\left(V_{r}^{\prime}\right)}{\mu\left(U_{r}\right)}=\lim _{r \rightarrow 0} \frac{m_{\varphi}\left(V_{r}^{\prime}\right)}{m_{\varphi}\left(U_{r}\right)}=1-e^{S_{p} \varphi(z)} .
$$

We set $\tau_{r}^{\prime}:=\inf \left\{n \geqslant 1: f^{n}(x) \in V_{r}^{\prime}\right\}$. Now [FFT2, Proposition 2.7] (with $B=U_{r}$ and $A=V_{r}^{\prime}$ ) implies that

$$
\mu\left(\tau_{r}^{\prime}>n\right)-\mu\left(\tau_{r}>n\right) \leqslant \sum_{j=1}^{p} \mu\left(\tau_{r}^{\prime}>n \wedge f^{-n+j}\left(V_{r}\right)\right) \leqslant p \mu\left(V_{r}\right)<p \mu\left(U_{r}\right)
$$

for all large $n$. So we now proceed as in Remark 1.1 .

$$
\begin{aligned}
0 & \leqslant \frac{-\log \mu\left(\tau_{r}>t\right)}{s \mu\left(U_{r}\right)^{1-\alpha}}<\frac{-\log \left(\mu\left(\tau_{r}^{\prime}>t\right)-p \mu\left(U_{r}\right)\right)}{s \mu\left(U_{r}\right)^{1-\alpha}} \\
& =\frac{-\log \left(1-\mu\left(\tau_{r}^{\prime} \leqslant t\right)-p \mu\left(U_{r}\right)\right)}{s \mu\left(U_{r}\right)^{1-\alpha}}=\frac{-\log \left(1-\mu\left(\cup_{j=0}^{t-1} f^{-j}\left(V_{r}^{\prime}\right)\right)-p \mu\left(U_{r}\right)\right)}{s \mu\left(U_{r}\right)^{1-\alpha}} \\
& \leqslant \frac{-\log \left(1-t \mu\left(V_{r}^{\prime}\right)-p \mu\left(U_{r}\right)\right)}{s \mu\left(U_{r}\right)^{1-\alpha}}=\frac{-\log \left(1-s \mu\left(V_{r}^{\prime}\right) \mu\left(U_{r}\right)^{-\alpha}-p \mu\left(U_{r}\right)\right)}{s \mu\left(U_{r}\right)^{1-\alpha}} .
\end{aligned}
$$

So by (3.8), the upper bound above converges to $1-e^{S_{p} \varphi(z)}$ as $\mu\left(U_{r}\right) \rightarrow 0$, so we conclude that $L_{\alpha, s}(z)=1-e^{S_{p} \varphi(z)}$.

Case II: $\alpha \in(\mathbf{1}, \infty)$.

For $\alpha>1$, we obtain the following upper bound:

$$
\begin{aligned}
-\frac{\log \mu_{Y}\left(\tau_{r}>t\right)}{s \mu\left(U_{r}\right)^{1-\alpha}} & =\frac{\log \mu(Y)}{s \mu\left(U_{r}\right)^{1-\alpha}}-\frac{\log \mu\left(\tau_{r}>t \wedge Y\right)}{s \mu\left(U_{r}\right)^{1-\alpha}} \\
& \sim-\frac{\log \mu\left(\tau_{r}>t \wedge Y\right)}{s \mu\left(U_{r}\right)^{1-\alpha}} \geqslant-\frac{\log \mu\left(\tau_{r}>t\right)}{s \mu\left(U_{r}\right)^{1-\alpha}} .
\end{aligned}
$$


So we conclude that $L_{\alpha, s}(z) \leqslant L_{Y, \alpha, s}(z)$. Note the above shows $L_{Y, \alpha, s}(z)=0$ implies $L_{\alpha, s}(z)=0$ so that items (3) and (4) of the theorem hold for $\alpha>1$.

To prove items (1) and (2) of the theorem, we also need a lower bound on $L_{\alpha, s}(z)$. For this, recall that the measure $\mu$ can be expressed in terms of $\mu_{Y}$ by,

$$
\mu(A)=\frac{1}{\int R_{Y} d \mu_{Y}} \sum_{k=0}^{\infty} \sum_{i=0}^{k} \mu_{Y}\left(f^{-i}(A) \cap Y_{k}\right),
$$

where $Y_{k}=\left\{R_{Y}=k\right\}$, and $A$ is any measurable set. Applying this expression to $A=\left\{\tau_{r}>t\right\}$, we note that $f^{-i}\left(\tau_{r}>t\right) \cap Y_{k}=\left\{\tau_{r}>t+i\right\} \cap Y_{k}$ since $U_{r} \subset Y$. Then reversing order of summation, we obtain,

$$
\mu\left(\tau_{r}>t\right)=\frac{1}{\int R_{Y} d \mu_{Y}} \sum_{i=0}^{\infty} \sum_{k=i}^{\infty} \mu_{Y}\left(\tau_{r}>t+i \wedge Y_{k}\right) \leqslant \mu(Y) \sum_{i=0}^{\infty} \mu_{Y}\left(\tau_{r}>t+i\right) .
$$

To proceed, we prove a slight extension of our estimates in Section 2.5. Let $\stackrel{\circ}{\mathcal{L}}_{r}$ denote the punctured transfer operator for $F$ with potential $\Phi=S_{R_{Y}} \varphi$ and hole $U_{r}$ as defined in (2.3). By assumption on $F$ and Corollary 2.7, $\mathcal{L}_{r}^{\circ}=\Lambda_{r} \Pi_{r}+R_{r}$ has a uniform spectral gap, i.e., there exists $\beta>0$ such that the spectral radius of $\Lambda_{r}^{-1} R_{r}$ is less than $e^{-\beta}$ for all $r$ sufficiently small.

Lemma 3.6. For all $r>0$ sufficiently small and any $n \in \mathbb{N}$ such that $e^{-\beta n}<\mu_{Y}\left(U_{r}\right) \log \mu_{Y}\left(U_{r}\right)$, we have

$$
\mu_{Y}\left(\tau_{Y, r}>n\right)=\Lambda_{r}^{n}\left[1+\mathcal{O}\left(\mu_{Y}\left(U_{r}\right) \log \mu_{Y}\left(U_{r}\right)\right)\right]
$$

Proof. Noting that 2.8 is valid for all iterates of $F$, we write

$$
\mu\left(\tau_{Y, r}>n\right)=\Lambda_{r}^{n}\left[1+\int_{Y} \Lambda_{r}^{-n} \dot{\mathcal{L}}_{r}^{n}\left(g_{0}-g_{r}\right) d m\right],
$$

where $g_{0}$ and $g_{r}$ are the normalised eigenfunctions for $\mathcal{L}_{0}$ and $\stackrel{\circ}{\mathcal{L}}_{r}$, respectively. Following $(2.9)$, we note that the error term above can be split into two terms, one bounded by $C e^{-\beta n}$ and the other by $-C \mu_{Y}\left(U_{r}\right) \log \mu_{Y}\left(U_{r}\right)$. By assumption on $n$, the error is of order $\mu_{Y}\left(U_{r}\right) \log \mu_{Y}\left(U_{r}\right)$.

Now fix $\varepsilon>0$ and define $A_{u}=A_{Y, u, \varepsilon}$ as before. Recall that if $\tau_{r}(y)>n$ and $y \in A_{n \mu(Y)}^{c}$, then $\tau_{Y, r}>n \mu(Y) /(1+\varepsilon \mu(Y))$. We assume that there exist $C, c(\varepsilon), \gamma>0$ such that $\mu_{Y}\left(A_{u}\right) \leqslant$ $C e^{-c(\varepsilon) u^{\gamma}}$, and require that $\alpha<\frac{1}{1-\gamma}$.

For the sake of brevity, set $\vartheta=L_{Y, \alpha, s}(z)$, and by (2.5), we may choose $r$ sufficiently small so that $\Lambda_{r} \leqslant e^{-(1-\varepsilon) \mu_{Y}\left(U_{r}\right) \vartheta}$. Setting $n=t+i, \rho_{r}=\mu_{Y}\left(U_{r}\right) \log \mu_{Y}\left(U_{r}\right)$, and using Lemma 3.6, we estimate each term in 3.9 by

$$
\begin{aligned}
\mu_{Y}\left(\tau_{r}>t+i\right) & \leqslant \mu_{Y}\left(\tau_{r}>t+i \wedge A_{(t+i) \mu(Y)}^{c}\right)+\mu_{Y}\left(A_{(t+i) \mu(Y)}\right) \\
& \leqslant \mu_{Y}\left(\tau_{Y, r}>(t+i) \mu(Y) /(1+\varepsilon \mu(Y))\right)+\mu_{Y}\left(A_{(t+i) \mu(Y)}\right) \\
& \leqslant \Lambda_{r}^{(t+i) \mu(Y) /(1+\varepsilon \mu(Y))}\left[1+\mathcal{O}\left(\rho_{r}\right)\right]+\mu_{Y}\left(A_{(t+i) \mu(Y)}\right) \\
& \leqslant e^{-(1-\varepsilon) \mu\left(U_{r}\right) \vartheta(t+i) /(1+\varepsilon \mu(Y))}\left[1+\mathcal{O}\left(\rho_{r}\right)\right]+C e^{-c(\varepsilon)(t+i)^{\gamma} \mu(Y)^{\gamma}} .
\end{aligned}
$$


To estimate (3.9), we must sum both terms above over $i$. Recalling that $t=s \mu\left(U_{r}\right)^{-\alpha}$, the sum over the first term is bounded by,

$$
\begin{aligned}
\sum_{i \geqslant 0} e^{-(1-\varepsilon) \mu\left(U_{r}\right) \vartheta(t+i) /(1+\varepsilon \mu(Y))}\left[1+\mathcal{O}\left(\rho_{r}\right)\right] & =\frac{\left[1+\mathcal{O}\left(\rho_{r}\right)\right] e^{-(1-\varepsilon) \vartheta s \mu\left(U_{r}\right)^{1-\alpha} /(1+\varepsilon \mu(Y))}}{1-e^{-(1-\varepsilon) \vartheta \mu\left(U_{r}\right) /(1+\varepsilon \mu(Y))}} \\
& \leqslant \frac{2 e^{-(1-\varepsilon) \vartheta s \mu\left(U_{r}\right)^{1-\alpha} /(1+\varepsilon \mu(Y))}}{(1-\varepsilon) \vartheta \mu\left(U_{r}\right)}
\end{aligned}
$$

for $\varepsilon$ and $r$ sufficiently small. The sum over the second term is (recalling that $c=c(\varepsilon)$ ),

$$
\sum_{i \geqslant 0} C e^{-c(t+i)^{\gamma} \mu(Y)^{\gamma}} \leqslant C \int_{0}^{\infty} e^{-c(t+x)^{\gamma} \mu(Y)^{\gamma}} d x=\frac{C}{c^{1 / \gamma} \mu(Y) \gamma} \int_{c t^{\gamma} \mu(Y)^{\gamma}}^{\infty} e^{-y} y^{\frac{1}{\gamma}-1} d y,
$$

where we have changed variables, $y=c(t+x)^{\gamma} \mu(Y)^{\gamma}$. Setting $n=\left\lceil\frac{1}{\gamma}-1\right\rceil$, we have $y^{\frac{1}{\gamma}-1} \leqslant y^{n}$, so making this substitution and integrating by parts $n$ times, yields

$$
\sum_{i \geqslant 0} C e^{-c(t+i)^{\gamma} \mu(Y)^{\gamma}} \leqslant \frac{C e^{-c t^{\gamma} \mu(Y)^{\gamma}}}{c^{1 / \gamma} \mu(Y) \gamma} \sum_{k=0}^{n} \frac{n !}{k !}\left(c t^{\gamma} \mu(Y)^{\gamma}\right)^{n-k} \leqslant \frac{e C n ! t e^{-c t^{\gamma} \mu(Y)^{\gamma}}}{\gamma c^{\frac{1}{\gamma}-n} \mu(Y)^{1-\gamma n}} .
$$

Putting these estimates together with (3.9), we have,

$$
\mu\left(\tau_{r}>t\right) \leqslant \mu(Y)\left[\frac{2 e^{-(1-\varepsilon) \vartheta s \mu\left(U_{r}\right)^{1-\alpha} /(1+\varepsilon \mu(Y))}}{(1-\varepsilon) \vartheta \mu\left(U_{r}\right)}+C^{\prime} t e^{-c(s \mu(Y))^{\gamma} \mu\left(U_{r}\right)^{-\alpha \gamma}}\right],
$$

so that

$$
-\log \mu\left(\tau_{r}>t\right) \geqslant \log \left(\frac{(1-\varepsilon) \vartheta \mu\left(U_{r}\right)}{2 \mu(Y)}\right)+\frac{(1-\varepsilon) \vartheta s \mu\left(U_{r}\right)^{1-\alpha}}{1+\varepsilon \mu(Y)}-\log \left[1+B_{r}\right],
$$

where

$$
B_{r}=\frac{C^{\prime} s \mu\left(U_{r}\right)^{1-\alpha}(1-\varepsilon) \vartheta}{2} e^{-c(s \mu(Y))^{\gamma} \mu\left(U_{r}\right)^{-\alpha \gamma}+(1-\varepsilon) \vartheta s \mu\left(U_{r}\right)^{1-\alpha} /(1+\varepsilon \mu(Y))} .
$$

Note that $B_{r} \rightarrow 0$ as $r \rightarrow 0$ since $\alpha<\frac{1}{1-\gamma}$. Thus dividing by $s \mu\left(U_{r}\right)^{1-\alpha}$ and recalling that $\alpha>1$, we have,

$$
\lim _{r \rightarrow 0} \frac{-\log \mu\left(\tau_{r}>t\right)}{s \mu\left(U_{r}\right)^{1-\alpha}} \geqslant \vartheta \frac{1-\varepsilon}{1+\varepsilon \mu(Y)},
$$

which is the required lower bound since $\varepsilon>0$ was arbitrary. Thus $L_{\alpha, s}(z)=L_{Y, \alpha, s}(z)$ and items (1) and (2) of the theorem are proved for this case.

Case III: $\alpha=\infty$. First we note that an upper bound similar to the one derived in Case II holds:

$$
\begin{aligned}
\lim _{t \rightarrow \infty}-\frac{1}{t} \log \mu_{Y}\left(\tau_{r}>t\right) & =\lim _{t \rightarrow \infty} \frac{1}{t} \log \mu(Y)-\frac{1}{t} \log \mu\left(\tau_{r}>t \wedge Y\right) \\
& \geqslant \lim _{t \rightarrow \infty}-\frac{1}{t} \log \mu\left(\tau_{r}>t\right) .
\end{aligned}
$$

To prove items (2)-(4) of the Theorem, we must show $L_{\infty, s}(z)=0$ under the assumption $\mu_{Y}\left(R_{Y} \geqslant t\right) \geqslant C e^{c t \gamma}$ for some $\gamma \in(0,1)$. This follows from Case III in the proof of Proposition 3.5 since then $L_{Y, \infty, s}(z)=0$. Due to the upper bound above, $L_{\infty, s}(z)=0$ as well. 
To prove item (1) of the theorem, fix $\varepsilon>0$ and assume there exists $c(\varepsilon)>0$ such that $\mu_{Y}\left(A_{u}\right) \leqslant$ $C e^{-c(\varepsilon) u}$. As in Case II, we take $r_{0}$ so small that the transfer operators $\dot{\mathcal{L}}_{r}$ associated with the induced map $F$ have a uniform spectral gap for all $r \in\left[0, r_{0}\right]$ and denote their leading eigenvalues by $\Lambda_{r}$. Using $(2.5)$, we choose $r_{1}<r_{0}$ so small that $e^{-(1-\varepsilon) \mu_{Y}\left(U_{r}\right) \vartheta} \geqslant \Lambda_{r} \geqslant e^{-c(\varepsilon)(1+\varepsilon \mu(Y)) / 2}$ for all $r<r_{1}$.

By (3.6) in the proof of Proposition 3.5 (1), and 3.10), we have

$$
\lim _{t \rightarrow \infty}-\frac{1}{t} \log \mu\left(\tau_{r}>t\right) \leqslant-\mu(Y) \log \Lambda_{r}
$$

To prove the corresponding lower bound, we follow (3.9) and the estimates in Case II of the present proof (with $\gamma=1$ ). In particular, using Lemma|3.6.

$$
\begin{aligned}
\mu_{Y}\left(\tau_{r}>t+i\right) & \leqslant \mu_{Y}\left(\tau_{r}>t+i \wedge A_{(t+i) \mu(Y)}^{c}\right)+\mu_{Y}\left(A_{(t+i) \mu(Y)}\right) \\
& \leqslant \Lambda_{r}^{(t+i) \mu(Y) /(1+\varepsilon \mu(Y))}\left[1+\mathcal{O}\left(\rho_{r}\right)\right]+C e^{-c(\varepsilon)(t+i) \mu(Y)} .
\end{aligned}
$$

Summing over $i$, we obtain

$$
\mu\left(\tau_{r}>t\right) \leqslant \mu(Y)\left[\frac{2 \Lambda_{r}^{t \mu(Y) /(1+\varepsilon \mu(Y)}}{(1-\varepsilon) \vartheta \mu\left(U_{r}\right)}+C e^{-c \mu(Y) t}\right] .
$$

And finally,

$$
-\log \mu\left(\tau_{r}>t\right) \geqslant \log \frac{(1-\varepsilon) \vartheta \mu\left(U_{r}\right)}{2 \mu(Y)}-\frac{t \mu(Y) \log \Lambda_{r}}{1+\varepsilon \mu(Y)}-\log \left[1+B_{r}\right]
$$

where $B_{r} \leqslant \frac{C(1-\varepsilon) \vartheta \mu\left(U_{r}\right)}{2} e^{-c \mu(Y) t / 2}$, by choice of $r_{1}$. Now dividing by $t$ and taking $t \rightarrow \infty$ yields

$$
\lim _{t \rightarrow \infty}-\frac{1}{t} \log \mu\left(\tau_{r}>t\right) \geqslant-\frac{\mu(Y) \log \Lambda_{r}}{1+\varepsilon \mu(Y)} .
$$

Since $\varepsilon>0$ was arbitrary, our upper and lower bounds match. Thus using again (2.5), we have

$$
\lim _{r \rightarrow 0} \lim _{t \rightarrow \infty} \frac{-\log \mu\left(\tau_{r}>t\right)}{t \mu\left(U_{r}\right)}=\lim _{r \rightarrow 0} \frac{-\mu(Y) \log \Lambda_{r}}{\mu\left(U_{r}\right)}=\lim _{r \rightarrow 0} \frac{-\log \Lambda_{r}}{\mu_{Y}\left(U_{r}\right)}=1
$$

in the generic case, and $1-e^{S_{p} \varphi(z)}$ in the periodic case. Thus $L_{\infty, s}(z)=L_{Y, \infty, s}(z)$ as required.

3.2. Remarks about the Polynomial Case. Theorem 3.2 gives optimal results when the induced system has deviations that are superpolynomial and when the decay rate of $A_{u}$ matches that of $\left\{R_{Y} \geqslant u\right\}$. However, it gives only partial results if the induced system has only polynomial deviations, i.e., $\mu_{Y}\left(A_{u}\right) \approx u^{-\beta}$ and $\mu_{Y}\left(R_{Y} \geqslant u\right) \geqslant u^{-\beta-1}$. In particular, the proofs of Proposition 3.5(4) and Theorem 3.2(4) yields in the generic case,

$$
L_{\alpha, s}(z)=L_{Y, \alpha, s}(z)=0 \text { if } \alpha>1 \text {, and } L_{\alpha, s}(z)=L_{Y, \alpha, s}(z)=1 \text { if } \frac{1}{1+\beta}<\alpha \leqslant 1 .
$$

It might appear that by improving our upper and lower bounds in (3.3) and (3.4), we might extend our results to the case $\alpha \leqslant 1 /(1+\beta)$, but a closer look reveals there is a real obstruction to using inducing arguments to evaluate the required limits in the polynomial case. In particular, there is a nontrivial dependence between the sets $\left\{\tau_{r}>t\right\}$ and $A_{u}$ which makes the polynomial case particularly delicate from this point of view. 
To illustrate this point, consider the class of Manneville-Pomeau or LSV maps on the unit interval, defined by

$$
f(x)= \begin{cases}x+2^{\gamma} x^{1+\gamma}, & \text { for } x \in[0,1 / 2) \\ 2 x-1, & \text { for } x \in[1 / 2,1]\end{cases}
$$

When $\gamma \in(0,1)$, these maps preserve an invariant probability measure $\mu$, absolutely continuous with respect to Lebesgue, with density $g \approx x^{-\gamma}$ for $x$ near 0 [Y, LSV2].

Set $Y=[1 / 2,1]$ and let $\left(U_{r}\right)_{r \in\left(0, r_{0}\right]} \subset(1 / 2+\delta, 1)$, for some $\delta>0$. For $k \geqslant 0$, let $a_{k}=f_{L}^{-k}(1 / 2)$, where $f_{L}$ is the left branch of $f$. Set $J_{0}=Y$ and $J_{k}=\left[a_{k}, a_{k-1}\right)$ for $k \geqslant 1$. Note that $\tau_{Y}=k+1$ on $f_{R}^{-1}\left(J_{k}\right)$, where $f_{R}$ is the right branch of $f$.

We claim $\left\{\tau_{r}>t \wedge A_{u}\right\} \supseteq \bigcup_{k \geqslant t} f_{R}^{-1}\left(J_{k}\right)$. Note that $\bigcup_{k \geqslant t} f_{R}^{-1}\left(J_{k}\right)=\left\{R_{Y}>t\right\}$, and that $\left\{\tau_{r}>t\right\} \supset\left\{R_{Y}>t\right\}$ since $U_{r} \subset Y$. Moreover, if $R_{Y}(x)>t$, then $\tau_{Y, u}(x)>u-1+t$, and for $u=\mu(Y) t$, we have

$$
\frac{1}{u} \tau_{Y, u}(x)>1-\frac{1}{u}+\frac{1}{\mu(Y)} \Longrightarrow \frac{1}{u} \tau_{Y, u}(x)-\frac{1}{\mu(Y)}>1-\frac{1}{u},
$$

so that $x \in A_{u}$ for all $u \geqslant 2$ and $\varepsilon<1 / 2$. Thus $\left\{R_{Y}>t\right\} \subset A_{u}$, completing the proof of the claim.

Using well-known estimates [LSV2] on the spacing of $a_{k}, a_{k} \approx k^{-1 / \gamma}$,

$$
\mu_{Y}\left(\tau_{r}>t \wedge A_{u}\right) \geqslant c t^{-1 / \gamma}=c s^{-1 / \gamma} \mu\left(U_{r}\right)^{\alpha / \gamma}
$$

for some uniform constant $c>0$, where as usual we have set $t=s \mu\left(U_{r}\right)^{-\alpha}$. Using this lower bound, we may split up the relevant expression in the limit defining $L_{Y, \alpha, s}$ as follows,

$$
\frac{-\log \mu_{Y}\left(\tau_{r}>t\right)}{s \mu\left(U_{r}\right)^{1-\alpha}}=\frac{-\log \mu_{Y}\left(\tau_{r}>t \wedge A_{u}^{c}\right)}{s \mu\left(U_{r}\right)^{1-\alpha}}-\frac{\log \left[1+\frac{\mu_{Y}\left(\tau_{r}>t \wedge A_{u}\right)}{\mu_{Y}\left(\tau_{r}>t \wedge A_{u}^{c}\right)}\right]}{s \mu\left(U_{r}\right)^{1-\alpha}} .
$$

To use the results for the induced map, one would expect that the first term above tends to the desired limit, while the second term above acts as an error term and tends to 0 as $r \rightarrow 0$. However, using (3.11), we see that the 'error' term is bounded below by

$$
\frac{\mu_{Y}\left(\tau_{r}>t \wedge A_{u}\right)}{s \mu\left(U_{r}\right)^{1-\alpha}} \geqslant c^{\prime} \mu\left(U_{r}\right)^{-1+\alpha+\alpha / \gamma} \underset{r \rightarrow 0}{\longrightarrow} \infty,
$$

whenever $\alpha<\gamma /(1+\gamma)$.

By Remark 1.1, we known all limit points of $L_{Y, \alpha, s}$ lie in $[0,1]$, so in the range $\alpha<\gamma /(1+\gamma)$, the limit relies on cancellation between two diverging terms in (3.12). This implies that what we would like to consider to be an error term does not function as one for small $\alpha$.

\section{Applications of inducing}

Theorem 3.2 applies whenever we have a system $(I, f, \mu)$ with an inducing scheme $(X, F, \nu)$ where $F=f^{\tau}$ and $\tau$ is the first return time to $X$ where, moreover, $\nu\left(A_{u}\right)$ is known to satisfy a suitable large deviations principle. At present such large deviations principles are known in quite specific cases. We mention several examples here. 
4.1. Generalised Farey maps. In the i.i.d. case it has been shown that the large deviation rate of an unbounded observable $\psi$ matches the tail of the observable. For example for $\bar{\psi}=\int \psi d \nu$, $\gamma \in(0,1)$ and $c>0$,

$$
\nu(\psi>n) \leqslant c e^{-n^{\gamma}} \Longrightarrow \lim _{n \rightarrow \infty} \frac{1}{n^{\gamma}} \log \nu\left(S_{n} \psi>\varepsilon+\bar{\psi}\right)=-\varepsilon^{\gamma},
$$

where $S_{n} \psi$ is the $n$-th ergodic sum of these observables, see [GRR. Similarly, if the tail of an observable is polynomial of order $\beta$, then the deviations are polynomial of order $\beta-1$; and for exponential, the orders match exactly [G].

An application of Theorem 3.2 is to generalised Farey maps as in [KMS. Here one chooses a countable partition $\left\{A_{n}\right\}_{n}$ of $(0,1]$ by left-open, right-closed intervals labelled in decreasing order in the interval with length of $A_{n}$ equal to $a_{n}$ for each $n$. Then for $t_{n}:=\sum_{k=n}^{\infty} a_{k}$ and $x \in[0,1]$,

$$
f(x)= \begin{cases}(1-x) / a_{1} & \text { if } x \in A_{1}, \\ a_{n-1}\left(x-t_{n+1}\right) / a_{n}+t_{n} & \text { if } x \in A_{n}, n \geqslant 2, \\ 0 & \text { if } x=0 .\end{cases}
$$

Lebesgue measure is invariant for this map and taking a first return map to the interval $A_{1}$ gives us an induced map satisfying the conditions of Theorem 2.5. Since the branches are linear, the map behaves in an i.i.d. manner so that Lebesgue measure is a Markov measure for the induced map.

Moreover, one can choose the intervals $\left\{A_{n}\right\}_{n}$ in such a way that any of the tail decay conditions given by $\left(t_{n}\right)_{n}$ apply to our observable $R_{Y}$. By the results above these match the large deviations, so we may also apply the appropriate items of Theorem 3.2. We observe that the only points $z$ which this theorem does not apply to directly are $\cup_{n \geqslant 0} f^{-n} 0$. It is straightforward to adapt the theorem slightly to cover all elements of this set except 0 .

4.2. Maps with exponential tails. If we start with an interval map $f: I \rightarrow I$ and can find a well-behaved first return map to an interval $Y \subset I$ with exponential tails, then Theorem 3.2(1) holds. That is, we require the first return map $F=f^{R_{Y}}$ to be a full-branched Gibbs-Markov map where the induced measure $\mu_{Y}$ has $\mu_{Y}\left(n-1 \leqslant R_{Y}<n\right) \leqslant C e^{-\beta n}$ for some constants $C, \beta>0$. By Section 2.6.2, F satisfies (F1)-(F4) of Section 2 .

The fact that a full-branched Gibbs-Markov map has exponential large deviations for observables with exponential tails appears to be essentially folklore. Yuri [Yu quotes such a result, but the setting is slightly different and the proof there is not given explicitly, so for completeness, we provide the proof in Appendix B. Since $R_{Y}$ has exponential tails, it follows from Proposition B.1 and Corollary B.2 that $R_{Y}$ satisfies a (local) exponential large deviations estimate and thus Theorem 3.2(1) applies to the original system $f: I \rightarrow I$.

We remark that by this argument, Theorem $3.2(1)$ applies to the tower map $\left(f_{\Delta}, \Delta\right)$ whenever one can construct a Young tower $[\mathrm{Y}]$ over an interval as described in the proof of Theorem 3.2 .

In order to develop a specific class of examples, for the remainder of this section, we make the following standing assumptions. We assume that $f: I \rightarrow I$ is a $C^{2}$, topologically mixing 
unimodal map with critical point $c$ and $\operatorname{orb}(c)=\left\{f^{n}(c): n \geqslant 1\right\}$ nowhere dense 4 Then one can find an interval $Y$, compactly contained in $I \backslash \overline{\operatorname{orb}(c)}$, such that $(Y, F)$ is full branched (see [MeS, Chapter 4] for details), where $F$ is the first return map to $Y$. Moreover we assume $F$ has bounded distortion, e.g. $f$ has negative Schwarzian derivative; then $(Y, F)$ is Gibbs-Markov. Finally, we assume that our measure is an equilibrium state for some $\varphi$ and discuss when our induced system has $(\mathrm{F} 1)-(\mathrm{F} 4)$ and the return time has exponential tails so that we can conclude that Theorem $3.2(1)$ holds.

4.2.1. Collet-Eckmann case. If $f$ satisfies the Collet-Eckmann condition (i.e., $\left|D f^{n}(c)\right|$ grows exponentially, and this case includes Misiurewicz maps, i.e., $c$ is not recurrent nor attracted to a stable periodic orbit, provided $f$ is non-flat at $c$ ), then for $\varphi=-t \log |D f|$, there is a unique equilibrium state for each $t$ in a neighbourhood of $[0,1]$, see for example [PR. Moreover, it can be deduced (e.g. from [PR]) that $(Y, F)$ satisfies the conditions (F1)-(F4) for Theorem 2.1 to hold for the induced version of $\mu_{t}$ (note that the conformal measure is w.r.t. the normalised potential $\varphi-P(\varphi)$ ), and that the return time has exponential tails. By Corollary B.2, $R_{Y}$ enjoys exponential large deviations with respect to the equilibrium measure $\mu_{t}$. Thus choosing $z \in Y_{\text {cont }}$ so that (U) is satisfied, it follows that Theorem 3.2(1) holds for each $t$ in a neighborhood of $[0,1]$.

4.2.2. Non-Collet Eckmann case. If $f$ fails the Collet-Eckmann condition, then for the potential $\varphi=-t \log |D f|$, there is still a unique equilibrium state for $t \in\left(t_{0}, 1\right)$ for some $t_{0}<0$, again see for example [PR]. Moreover, $(Y, F)$ satisfies the conditions for Theorem 2.1 to hold for the induced version of $\mu_{t}$, and the return time has exponential tails. So again choosing $z \in Y_{\text {cont }}$ so that (U) is satisfied, Theorem 3.2(1) holds for this class of potentials. By contrast, for $t=1$, even if there is an equilibrium state for $-\log |D f|$, it will have sub-exponential mixing, so Theorem 3.2(1) will fail.

4.2.3. Lipschitz potentials. If $\varphi$ is a Lipschitz potential, then our results hold more generally: for Theorem 3.2(1) to hold for the equilibrium state we only need the potential to be hyperbolic, i.e., $\sup _{x \in I} \frac{1}{n} S_{n} \varphi(x)<P(\varphi)$ for some $n$, where $P(\varphi)$ denotes the variational pressure. As shown in [LR] this is automatic if we merely assume that $\left|D f^{n}(f(c))\right| \rightarrow \infty$.

\section{Appendix A. Proof of Proposition 2.5}

The $L^{1}$ bound on $\dot{\mathcal{L}}_{r}^{n} \psi$ in Proposition 2.5 follows directly from $(2.4)$, so we focus on proving the required bound on the variation of $\stackrel{\circ}{\mathcal{L}}_{r}^{n} \psi$.

\footnotetext{
${ }^{4}$ We note that we can drop the topologically mixing and unimodal assumptions, but this makes our statements more involved. Similarly, one can also drop the requirement that orb $(c)$ be nowhere dense, see for example DT].
} 
For $r \in\left[0, r_{0}\right]$, let $\mathcal{I}_{r}^{n}=\left\{J_{i}\right\}_{i}=\left\{\left(a_{i}, b_{i}\right)\right\}$ denote the intervals of monotonicity for $f_{r}^{n}$ and set $K_{i}=f^{n}\left(J_{i}\right)$. Then for $\psi \in \mathcal{B}$ and $n \geqslant 0$, we estimate,

$$
\begin{aligned}
\bigvee_{I} \dot{\mathcal{L}}_{r}^{n} \psi & \leqslant \sum_{i} \bigvee_{J_{i}} \psi e^{S_{n} \varphi}+\psi\left(a_{i}\right) e^{S_{n} \varphi\left(a_{i}\right)}+\psi\left(b_{i}\right) e^{S_{n} \varphi\left(b_{i}\right)} \\
& \leqslant \sum_{i} 2 \bigvee_{J_{i}} \psi e^{S_{n} \varphi}+\frac{1}{m_{0}\left(J_{i}\right)} \int_{J_{i}} \psi e^{S_{n} \varphi} d m_{0}
\end{aligned}
$$

For the second term in A.1, we note that by conformality and the bounded distortion property (F1), we have for each $x \in J_{i}$,

$$
e^{S_{n} \varphi(x)} \cdot \frac{m_{0}\left(K_{i}\right)}{m_{0}\left(J_{i}\right)} \leqslant 1+C_{d}
$$

For the first term in A.1, we split

$$
\bigvee_{J_{i}} \psi e^{S_{n} \varphi} \leqslant \sup _{J_{i}} e^{S_{n} \varphi} \bigvee_{J_{i}} \psi+\sup _{J_{i}}|\psi| \bigvee_{J_{i}} e^{S_{n} \varphi} \leqslant \sup _{J_{i}} e^{S_{n} \varphi} \bigvee_{J_{i}} \psi+\sup _{J_{i}}|\psi| C_{d} \sup _{J_{i}} e^{S_{n} \varphi}
$$

where we have used Lemma $2.4(\mathrm{~b})$ to bound $\bigvee_{J_{i}} e^{S_{n} \varphi}$. Using the bound $\sup _{J_{i}}|\psi| \leqslant \bigvee_{J_{i}} \psi+$ $\left(m_{0}\left(J_{i}\right)\right)^{-1} \int_{J_{i}}|\psi| d m_{0}$, we put these estimates together in A.1 and use A.2 to obtain,

$$
\begin{aligned}
\bigvee_{I} \dot{\mathcal{L}}_{r}^{n} \psi & \leqslant \sum_{i}\left(2+2 C_{d}\right) \sup _{J_{i}} e^{S_{n} \varphi} \bigvee_{J_{i}} \psi+\frac{\left(1+C_{d}\right)\left(1+2 C_{d}\right)}{m_{0}\left(K_{i}\right)} \int_{J_{i}}|\psi| d m_{0} \\
& \leqslant\left(2+2 C_{d}\right)\left|e^{S_{n} \varphi}\right|_{\infty} \bigvee_{I} \psi+\inf _{i} \frac{\left(1+C_{d}\right)\left(1+2 C_{d}\right)}{m_{0}\left(K_{i}\right)} \int_{I_{r}^{n-1}}|\psi| d m_{0} .
\end{aligned}
$$

Applying A.4 when $n=n_{1}$, setting $\bar{\sigma}:=\left(2+2 C_{d}\right)\left|e^{S_{n_{1}} \varphi}\right|_{\infty}<1$, and using (U) yields,

$$
\bigvee_{I} \dot{\mathcal{L}}_{r}^{n_{1}} \psi \leqslant \bar{\sigma} \bigvee_{I} \psi+\frac{\left(1+2 C_{d}\right)^{2}}{c_{0}} \int_{\dot{I}_{r}^{n-1}}|\psi| d m_{0},
$$

and since $\bigvee_{I} e^{S_{n} \varphi}<\infty$ for each $n$, this relation can be iterated to complete the proof of Proposition 2.5 with $\sigma=\bar{\sigma}^{1 / n_{1}}$.

\section{Appendix B. Exponential Deviations}

In this section, we prove the fact that full-branched Gibbs-Markov maps have exponential large deviations for observables with exponential tails.

Let $P_{G}(\phi)$ denote the Gurevich pressure of $\phi$ (see [S1]). Note that as in [S1, Theorem 2], this is equal to the variational definition of pressure given in Section 2 .

Proposition B.1. Let $F$ be a full-branched Gibbs-Markov map and $\phi, \psi$ weakly Hölder continuous potentials. If there exists $\delta>0$ such that $P_{G}(\phi+t \psi)<\infty$ for each $|t|<\delta$ (or equivalently that $\left.\left|\mathcal{L}_{\phi+t \psi} 1\right|_{\infty}<\infty\right)$, then $\psi$ enjoys exponential large deviations for $\mu$ the equilibrium state for $\phi$.

Proof. First we note that the assumptions on $\phi$ imply: (i) $\phi$ has finite Gurevich pressure $P_{G}(\phi)$ [S1, Theorem 1]; (ii) $\phi$ is positive recurrent [S3, Corollary 2]; and (iii) there exists a finite 
conformal Borel measure $m_{\phi}$, positive on cylinders, such that $\frac{d m}{d m \circ F}=e^{\phi-P_{G}(\phi)}$ [S1, Theorem 4, Proposition 3].

Under these conditions, the associated transfer operator $\mathcal{L}_{\phi}$ acting on the space of Hölder continuous function $\$^{5}$ has a spectral gap. It then follows from [CS, Theorem 2.1], that $\phi$ is strongly positive recurrent. (We refer the reader to [CS] for the relevant definition.)

Strong positive recurrence implies that if $\psi$ is a weakly Hölder continuous function such that $P_{G}(\phi+t \psi)<\infty$ for all $|t|<\delta$ and some $\delta>0$, then $t \mapsto P_{G}(\phi+t \psi)$ is analytic in $t$ [S2, Theorem 3]. Moreover, $\phi+t \psi$ is positive recurrent for each $|t|<\delta$ and has a Gibbs measure $\mu_{t}$ which is moreover the unique equilibrium state for $\phi+t \psi$. Denote by $\mu=\mu_{0}$ the Gibbs measure for $\phi$. Without loss of generality, in what follows we assume $P_{G}(\phi)=\mu(\psi)=0$ and that 0 is a local minimum for $t \mapsto P_{G}(\phi+t \psi)$.

Now define $J_{n}^{+}(\varepsilon)$ to be the collection of $n$-cylinders containing a point $x$ so that $S_{n} \psi(x)>n \varepsilon$; similarly, let $J_{n}^{-}(\varepsilon)$ denote the collection of $n$-cylinders containing $x$ such that $S_{n} \psi(x)<-n \varepsilon$. We first consider $J_{n}^{+}(\varepsilon)$. Since $\left.\frac{d}{d t} P_{G}(\phi+t \psi)\right|_{t=t_{0}}=\mu_{t_{0}}(\psi)$ for $\left|t_{0}\right|<\delta$, and by continuity of the derivative for $\varepsilon$ small enough we can find $q>0$ so that $\mu_{q}(\psi)=\varepsilon$.

Then strict convexity of pressure implies that $P_{G}(q \psi+\phi)-q \varepsilon<0$ (a slightly more sophisticated argument allows us to express this in terms of the Helmholtz free energy, but we do not require this here).

Let $\mathcal{P}_{n}$ denote the set of $n$-cylinders and for $C_{n}^{i} \in \mathcal{P}_{n}$, let $x_{n}^{i}$ be the fixed point of $F^{n}$ in $C_{n}^{i}$. So we compute, using the Gibbs property (here the constant $C$ covers the Gibbs constant and distortion constants):

$$
\begin{aligned}
\mu\left(S_{n} \psi>n \varepsilon\right) & \leqslant \sum_{C_{n}^{i} \in J_{n}^{+}(\varepsilon)} \mu\left(C_{n}^{i}\right) \leqslant C \sum_{C_{n}^{i} \in J_{n}^{+}(\varepsilon)} e^{S_{n} \phi\left(x_{n}^{i}\right)} \\
& \leqslant C^{2} \sum_{C_{n}^{i} \in J_{n}^{+}(\varepsilon)} e^{\left.q\left(S_{n}(\psi-\varepsilon)\right)\left(x_{n}^{i}\right)\right)+S_{n} \phi\left(x_{n}^{i}\right)} \\
& \leqslant C^{2} e^{-n q \varepsilon} \sum_{C_{n}^{i} \in \mathcal{P}_{n}} e^{S_{n}\left(\phi+q \psi\left(x_{n}^{i}\right)\right)}
\end{aligned}
$$

Taking logarithms, dividing by $n$ and taking limits we obtain

$$
\limsup _{n \rightarrow \infty} \frac{1}{n} \log \mu\left(S_{n} \psi>n \varepsilon\right) \leqslant P(\phi+q \psi)-q \varepsilon<0
$$

as required. A similar argument, with $q<0$, applies to $J_{n}^{-}(\varepsilon)$.

Corollary B.2. Under the assumptions of Proposition B.1, suppose that $\psi$ is weakly Hölder continuous with exponential tails, i.e., $\mu(n-1<|\psi| \leqslant n) \leqslant e^{-\beta n}$, for some $\beta>0$. Then $\psi$ enjoys exponential local large deviations with respect to $\mu$.

\footnotetext{
${ }^{5}$ Hölder continuity here is defined using the same constant $\theta$ as for the potential $\phi$, i.e., weak Hölder continuity of $\phi$ means $\sup _{C_{n}^{i} \in \mathcal{P}_{n}} \sup \left\{|\phi(x)-\phi(y)|: x, y \in C_{n}^{i}\right\} \leqslant \theta^{n}$, where $\mathcal{P}_{n}$ is the set of $n$-cylinders for $F$. We study the transfer operator on the class of functions $f: X \rightarrow \mathbb{R}$ sharing the same property as $\phi$.
} 
Proof. Letting $\left\{x_{j}\right\}_{j}$ be the collection of all fixed points of $F$, and $\psi_{i}$ be the maximum value $|\psi|$ takes on the 1-cylinder $X_{i}$, by the Gibbs property,

$$
\begin{aligned}
\sum_{j} e^{(\phi+t \psi)\left(x_{j}\right)} & =\sum_{n \geqslant 1} \sum_{n-1<\psi_{j} \leqslant n} e^{(\phi+t \psi)\left(x_{j}\right)} \leqslant C^{2} \sum_{n} e^{n|t|} \mu(n-1<|\psi| \leqslant n) \\
& \leqslant C^{2} \sum_{n} e^{n(|t|-\beta)}<\infty
\end{aligned}
$$

provided $|t|<\beta$. Standard theory shows that this implies that $P_{G}(\phi+t \psi)<\infty$, so that $\psi$ satisfies the hypotheses of Proposition B.1.

\section{REFERENCES}

[AFV] H. Aytaç, J.M. Freitas, S. Vaienti, Laws of rare events for deterministic and random dynamical systems, Trans. Amer. Math. Soc. 367 (2015), 8229-8278.

[BT] H. Bruin, M. Todd, Return time statistics of invariant measures for interval maps with positive Lyapunov exponent, Stoch. Dyn. 9 (2009), 81-100.

[BY] L. Bunimovich, A. Yurchenko, Where to place a hole to achieve a maximal escape rate, Israel J. of Math. 182 (2011), 229-252.

[CS] V. Cyr, O. Sarig, Spectral gap and transience for Ruelle operators on countable Markov shifts, Commun. Math. Phys.292 (2009), 637-666.

[DF] M.F. Demers, B. Fernandez, Escape rates and singular limiting distributions for intermittent maps with holes, Trans. Amer. Math. Soc. 368 (2016), 4907-4932.

[DT] M.F. Demers, M. Todd, Equilibrium states, pressure and escape for multimodal maps with holes, Israel J. Math. 221 (2017), 367-424.

[FP] A. Ferguson, M. Pollicott, Escape rates for Gibbs measures, Ergodic Theory Dynam. Systems, 32 (2012), 961-988.

[FFT1] A.C. Freitas, J.M. Freitas, M. Todd, The compound Poisson limit ruling periodic extreme behaviour of non-uniformly hyperbolic dynamics, Comm. Math. Phys. 321 (2013), 483-527.

[FFT2] A.C. Freitas, J.M. Freitas, M. Todd, Speed of convergence for laws of rare events and escape rates, Stochastic Process. Appl. 125 (2015), 1653-1687.

[G] N. Gantert, Large deviations for a heavy-tailed mixing sequence, Preprint TU Berlin.

[GRR] N. Gantert, K. Ramanan, F. Rembart, Large deviations for weighted sums of stretched exponential random variables, Electron. Commun. Probab. 19 (2014), 1-14.

[H] N. Haydn, Entry and return times distribution, Dyn. Syst. 28 (2013), 333-353.

[KL1] G. Keller, C. Liverani, Stability of the spectrum for transfer operators, Annali della Scuola Normale Superiore di Pisa, Classe di Scienze (4) Vol. XXVIII, (1999), 141-152.

[KL2] G. Keller, C. Liverani, Rare events, escape rates and quasistationarity: some exact formulae, Journal of Statistical Physics 135 (2009), 519-534.

[KMS] M. Kesseböhmer, S. Munday, B.O. Stratmann, Strong renewal theorems and Lyapunov spectra for $\alpha$-Farey and $\alpha$-Lüroth systems, Ergodic Theory Dynam. Systems 32 (2012), 989-1017.

[LR] H. Li, J. Rivera-Letelier, Equilibrium states of weakly hyperbolic one-dimensional maps for Hölder potentials, Comm. Math. Phys. 328 (2014) 397-419.

[LSV1] C. Liverani, B. Saussol, S. Vaienti, Conformal measure and decay of correlation for covering weighted systems, Ergodic Theory Dynam. Systems 18:6 (1998), 1399-1420.

[LSV2] C. Liverani, B. Saussol, S. Vaienti, A probabilistic approach to intermittency, Ergodic Theory Dynam. Systems 19 (1999), 671-685.

$\left[\mathrm{LF}^{+}\right]$V. Lucarini, D. Faranda, A.C. Freitas, J.M. Freitas, M. Holland, T. Kuna, M. Nicol, M. Todd, S. Vaienti, Extremes in Dynamical Systems, Pure and Applied Mathematics: A Wiley Series of Texts, Monographs, and Tracts, 2016, pp 312.

[MeS] W. de Melo, S. van Strien, One dimensional dynamics, Ergebnisse Series 25, Springer-Verlag, 1993.

[PU] M. Pollicott, M. Urbanski, Open Conformal Systems and Perturbations of Transfer Operators, to appear in Lecture Notes in Mathematics 2206, Springer. 
[PR] F. Przytycki, J. Rivera-Letelier, Nice inducing schemes and the thermodynamics of rational maps, Comm. Math. Phys. 301 (2011) 661-707.

[R] M. Rychlik, Bounded variation and invariant measures, Studia Math. LXXVI (1983), 69-80.

[S1] O. Sarig, Thermodynamic Formalism for Countable Markov Shifts, Ergodic Theory Dyn. Syst. 19 (1999), 1565-1593.

[S2] O. Sarig, Phase transitions for countable topological Markov shifts, Commun. Math. Phys. 217 (2001), $555-577$.

[S3] O. Sarig, Existence of Gibbs measures for countable Markov shifts, Proc. Amer. Math. Soc. 131 (2003), 1751-1758.

[Y] L.-S. Young, Recurrence times and rates of mixing, Israel J. Math. 110 (1999), 153-188.

[Yu] M. Yuri, Large deviations for countable to one Markov systems, Comm. Math. Phys. 258 (2005), 455-474.

Henk Bruin, Faculty of Mathematics, Vienna University, Oskar Morgensternplatz 1, Vienna 1090, Austria

E-mail address: henk.bruin@univie.ac.at

$U R L:$ http://www.mat.univie.ac.at/ bruin/

Mark F. Demers, Department of Mathematics, Fairfield University, Fairfield, CT 06824, USA

E-mail address: mdemers@fairfield.edu

$U R L:$ http://faculty.fairfield.edu/mdemers

Mike Todd, Mathematical Institute, University of St Andrews, North Haugh, St Andrews, KY16 9SS, SCOTLAND

E-mail address: m.todd@st-andrews.ac.uk

$U R L:$ http://www.mcs.st-and.ac.uk/ miket/ 